\title{
Copolyesters made from 1,4-butanediol, sebacic acid and D-glucose by melt and enzymatic polycondensation
}

\author{
Cristina Japu, ${ }^{a}$ Antxon Martínez de llarduya, ${ }^{a}$ Abdelilah Alla ${ }^{a}$ \\ Yi Jiang, ${ }^{\mathrm{b}}$ Katja Loos $^{\mathrm{b}}$ and Sebastián Muñoz-Guerra ${ }^{\mathrm{a}}$ \\ ${ }^{a}$ Universitat Politècnica de Catalunya, ETSEIB, Diagonal 647, 08028 Barcelona, Spain \\ bernike Institute for Advanced Materials, University of Groningen, \\ Nijenborgh 4, 9747 AG Groningen, The Netherlands
}

Corresponding author: sebastian.munoz@upc.edu

\begin{abstract}
Biotechnologically accessible 1,4-butanediol and vegetal oil-based diethyl sebacate were copolymerized with bicyclic acetalized D-glucose derivatives (Glux) by polycondensation both in the melt at high temperature and in solution at mild temperature mediated by polymer-supported Candida antarctica lipase B (CALB). Two series of random copolyesters ( $\mathrm{PB}_{\mathrm{x}} \mathrm{Glux}_{\mathrm{y}} \mathrm{Seb}$ and PBSeb $_{x} G_{l u x}$ ) were prepared differing in which D-glucose derivative (Glux diol or Glux diester) was used as comonomer. The three parent homopolyesters PBSeb, PBGlux and PGluxSeb were prepared as well. Both methods were found to be effective for polymerization although significant higher molecular weights were achieved by melt polycondensation. The thermal properties displayed by the copolyesters were largely dependent on composition and also on the functionality of the replacing Glux unit. The thermal stability of PBSeb was retained or even slightly increased after copolymerization with Glux whereas crystallinity and melting temperature were largely depressed. On the contrary, the glass-transition temperature noticeably increased with the content in Glux units. PGluxSeb distinguished in displaying both $T_{\mathrm{g}}$ and $T_{\mathrm{m}}$ higher than PBSeb because a different crystal structure is adopted by this homopolyester. The hydrolytic degradability of PBSeb in water was enhanced by copolymerization, in particular when biodegradation was assisted by lipases.
\end{abstract}




\section{Introduction}

The growing interest in the availability of new materials from renewable resources is leading to the development of a number of sustainable and biodegradable polymers that intend to replace those traditionally produced from fossil fuels. ${ }^{1-4}$ Aliphatic polyesters are a family of biodegradable or partially biodegradable polymers that can be processed into various forms such as films, fibers and injection-molded devices. ${ }^{5,6}$ Outstanding examples of this family are poly(butylene succinate) (PBS), poly(L-lactic acid) (PLA), poly(e-caprolactone) (PCL) and poly(hydroxy alkanoate)s (PHAs) among others. Some of these polyesters have found utility in the packaging sector and many of them have been intensively investigated for their potential as biomaterials suitable for the manufacture of temporal prostheses or tissue engineering scaffolds. ${ }^{7,8}$

In this work novel aliphatic copolyesters derived from the poly(butylene sebacate) (PBSeb) are described. PBSeb is a well-known aliphatic polyester that is commonly prepared by polycondensation of 1,4-butanediol and diethyl sebacate. From the synthetic point of view, the sebacate esters are preferred over short chain dicarboxylic aliphatic acid derivatives because intramolecular condensation reactions leading to cyclization is in this case conveniently overcome. However the presence of the octamethylene segment in the main chain of PBSeb confers to the polymer unduly high flexibility and therefore low transition temperatures and poor mechanical behavior. In fact, PBSeb has a $T_{\mathrm{g}}$ of $-62{ }^{\circ} \mathrm{C}$, which is a value too low as to allow its use in applications requiring a minimum material stiffness. ${ }^{9}$ This has motivated a number of copolymerization approaches addressed to improve the basic properties of PBSeb. ${ }^{10,11}$ Aromatic units have proven to be the most efficient in enhancing PBSeb properties but their use unavoidably involves a significant detriment to both renewability and biodegradability. ${ }^{12}$ Recently the carbohydrate-based aromatic compound 2,4furandicarboxylic acid (FDCA) has been used to replaced partially sebacic acid to generate copolyesters displaying higher transition temperatures. ${ }^{13}$ In the present study, 
rigid carbohydrate-based bicyclic units derived from D-glucose are incorporated in PBSeb replacing either the butylene or the sebacate units. Mixtures of 1,4-butanediol, diethyl sebacate, and the bicyclic di-O-methylene diacetal of either D-glucitol or dimethyl D-glucarate are polycondensated in the melt to afford two series of copolyesters, namely $\mathrm{PB}_{\mathrm{x}}$ Glux $\mathrm{Seb}$ and $\mathrm{PBSeb}_{\mathrm{x}} \mathrm{Glux}_{\mathrm{y}}$. In these acronyms, $\mathrm{x}$ and $\mathrm{y}$ denote the \%-molar content of the copolyesters in butylene (B), sebacate (Seb) and glucose derived units (Glux), the latter being either glucitylene or glucarate units depending on whether the glucitol or the dimethyl glucarate diacetal is the comonomer used.

The prevailing criterion at selecting the comonomers for building a new PBSeb copolyesters has been to retain the bio-based nature of the parent homopolyester. 1,4Butanediol is a very popular monomer that is extensively used in the synthesis of important aliphatic and aromatic polyesters. The traditional production of this alkanediol makes use of petrochemicals as raw material but recently new processes based on the direct fermentation of D-glucose or on hydrogenation of bio-succinic acid offer the opportunity to produce renewable 1,4-butanediol. ${ }^{14}$ Sebacic acid is an aliphatic dicarboxylic acid readily available from castor oil $^{15}$ that in the human body happens as an intermediate product of the $\omega$-oxidation of long chain monocarboxylic acids. The excellent biocompatibility exhibited by this diacid resulted in the approval of the Food and Drug Administration (FDA) of USA for its use in the manufacturing of biomaterials. ${ }^{16}$ Sebacic acid-based polyesters have found various applications both in the biomedical field as biodegradable delivery systems, ${ }^{17,18}$ and in the packaging industry as component of blends for films. ${ }^{19-21}$ The sugar-based comonomers used in this work are bicyclic 2,4:3,5-di-O-methylene diacetals with the chemical formulae depicted in Scheme I. The structure of these diacetals consists of a C6-segment backbone with four asymmetric carbons forming part of the two fused 1,3-dioxane rings and with two hydroxyl or methoxycarbonyl functions at the end positions. We have 
recently reported on the ability of this type of bicyclic compounds to confer stiffness to polyester chains and to increase therefore the $T_{\mathrm{g}}$ of aliphatic copolyesters. ${ }^{22}$

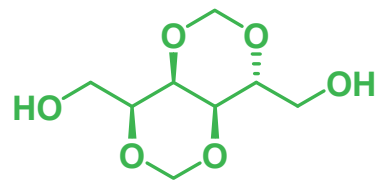

2,4:3,5-Di-O-methylene-D-glucitol (Glux-diol)

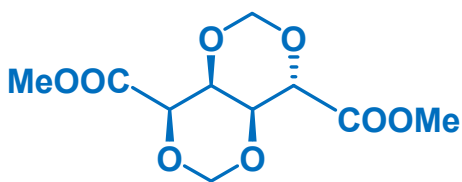

Dimethyl 2,4:3,5-di-O-methylene-D-glucarate (Glux-diester)

Scheme I. Chemical structures of bicyclic diacetals used for the synthesis of copolyesters.

Biodegradability is a distinguishing property of aliphatic polyesters that should be retained or even enhanced when modifications on the structure of the polymers are carried out with the purpose of improving other aspects of the material. The susceptibility of the polyester to biodegradation is enhanced when crystallinity is depressed and/or hydrophilicity is increased. The ability of Glux units to enhance the biodegradability of polyesters and to increase at the same time their glass-transition temperature $\left(T_{\mathrm{g}}\right)$ is a well proven fact that has been recently reported for a number of cases. $^{22}$ Other monocyclic and bicyclic diols and diacids derived from L-tartaric, ${ }^{23} \mathrm{D}$ galactose $^{24 a}$ and D-mannose ${ }^{24 b}$ are known to be able of exerting a similar effect on both aliphatic and aromatic polyesters.

The experience accumulated in the preparation of copolyesters containing sugar-based cyclic acetalized units makes more than reasonable to expect that the replacement of either the diol or the diacid units in PBSeb by the Glux ones leads to increase the $T_{\mathrm{g}}$ of the polyester without detriment of its biodegradability. Furthermore, given the bio-based origin of such comonomers, the sustainability of the polyester will be retained. Two polycondensation methods have been used in this work for the synthesis of these copolyesters, polycondensation in the melt under reaction conditions similar to those used for industrial production, and polycondensation in solution assisted by immobilized CALB. The enzyme mediated polycondensation is of particular 
interest in this case because milder conditions more compatible with the heat sensitivity of the sugar-based monomers can be used. Moreover the absence of metallic catalysts contributes to increase the sustainability grade of the synthesis. Several papers have been recently reported on the enzymatic synthesis of the homologous polyester poly(butylene succinate) ${ }^{25,26}$ but to our knowledge, this is the first time that the preparation of PBSeb is undertaken by this method.

\section{Experimental section}

\subsection{Materials and methods}

Materials. 2,4:3,5-Di-O-methylene-D-glucitol (Glux-diol) and dimethyl 2,4:3,5-di-Omethylene-D-glucarate (Glux-diester) were synthesized following the procedure recently reported by Japu et al. ${ }^{22}$ Diethyl sebacate (DES) (98\%), 1,4-butanediol (99\%), and the catalyst dibutyl tin oxide (DBTO, 98\%) were purchased from Sigma Aldrich. Irganox 1010 and Irgafos 126 antioxidants were generous gifts from BASF. Sodium trifluoroacetate (98\%) used to stabilize 1,1,1,3,3,3-hexafluoro-2-propanol (HFIP) and lipase from porcine pancreas (triacylglycerol lipase activity: 15-35 units/mg) were also purchased from Sigma-Aldrich. The solvents used for polycondensation, purification and characterization, such as diphenyl ether (DPO), chloroform, trifluoroacetic acid (TFA), methanol, diethyl ether, dichloracetic acid (DCA) were of high purity grade and used as received. Lipase acrylic resin from Candida antarctica (CALB) was purchased from Sigma Aldrich and it was dried in the presence of phosphorus pentoxide under vacuum for $48 \mathrm{~h}$ previous to use.

Methods. Intrinsic viscosities were measured in DCA using an Ubbelohde microviscometer thermostated at $25 \pm 0.1{ }^{\circ} \mathrm{C}$ using polymer solution concentrations ranging from 0.3 to $1 \mathrm{~g} \cdot \mathrm{dL}^{-1}$. Molecular weights were determined by GPC using a Waters GPC equipment provided with a RI detector. HFIP containing sodium trifluoroacetate $\left(6.8 \mathrm{~g} \cdot \mathrm{L}^{-1}\right)$ was the mobile phase. $100 \mu \mathrm{L}$ of $0.1 \%(\mathrm{w} / \mathrm{v})$ sample solution were injected and chromatographed under a flow of $0.5 \mathrm{~mL} \cdot \mathrm{min}^{-1}$. PL HFIPgel $300 \mathrm{x}$ 
$7.5 \mathrm{~mm}$ column and protected with a pre-column was used at $35^{\circ} \mathrm{C}$. Molar mass averages and their distributions were calculated against PMMA standards.

${ }^{1} \mathrm{H}$ and ${ }^{13} \mathrm{C}$ NMR spectra were recorded at $25^{\circ} \mathrm{C}$ using a Bruker AMX-300 spectrometer operating at 300.1 and $75.5 \mathrm{MHz}$, respectively. The spectra were internally referenced with tetramethylsilane. Samples were prepared by dissolving the polymer in deuterated chloroform. 10 and $50 \mathrm{mg}$ of sample in $1 \mathrm{~mL}$ of solvent were used for ${ }^{1} \mathrm{H}$ and ${ }^{13} \mathrm{C}$ NMR analyses, respectively. Sixty-four scans were recorded for ${ }^{1} \mathrm{H}$, and between 5,000 and 15,000 scans were collected for ${ }^{13} \mathrm{C}$ with 32 and $64 \mathrm{~K}$ data points and relaxation delays of 1 and $2 \mathrm{~s}$, respectively. Two-dimensional spectra (2D) as ${ }^{1} \mathrm{H}-{ }^{1} \mathrm{H}$ homonuclear (COSY) and ${ }^{13} \mathrm{C}-{ }^{1} \mathrm{H}$ heteronuclear shift correlation (HETCOR) were respectively recorded by means of the cosy and $h x c o$ pulse sequences implemented in the Bruker NMR instrument package.

Thermogravimetric analysis was carried out in a nitrogen atmosphere with a Perkin-Elmer TGA 6 thermobalance. Polymer samples with an approximate mass of 10 mg were analyzed in the $30-600{ }^{\circ} \mathrm{C}$ temperature range at a heating rate of $10^{\circ} \mathrm{C} \cdot \mathrm{min}^{-1}$. The thermal behavior of the polymers was examined by differential scanning calorimetry (DSC) using a Perkin-Elmer Pyris 1 and a Mettler 822 apparatus. Thermograms were obtained from 4-6 $\mathrm{mg}$ samples under a nitrogen flow of $20 \mathrm{~mL} \cdot \mathrm{min}^{-}$ 1. Indium and zinc were used as standards for temperature and enthalpy calibrations. The melting temperature $\left(T_{\mathrm{m}}\right)$ was taken as the maximum of the endothermic peak appearing on heating traces recorded at $10^{\circ} \mathrm{C} \cdot \mathrm{min}^{-1}$. The glass transition temperature $\left(T_{\mathrm{g}}\right)$ was determined from samples quenched from the melt as the inflection point seen on heating traces either recorded at $20^{\circ} \mathrm{C} \cdot \mathrm{min}^{-1}$ or by dynamic differential scanning calorimetry (DDSC) at modulated temperature. In this case a cyclic heating/cooling process oscillating between 4 and $2{ }^{\circ} \mathrm{C} \cdot \mathrm{min}^{-1}$ was applied to the sample by using the DDSC program implemented on the Pyris 1 equipment.

X-ray diffraction profiles were recorded on the PANalytical X'Pert PRO MPD $\theta / \theta$ diffractometer using the $\mathrm{Cu}-\mathrm{Ka}$ radiation of wavelength $0.1542 \mathrm{~nm}$ from powder 
samples coming directly from synthesis. Scanning Electron Microscopy (SEM) images were taken with a field-emission JEOL JSM-7001F instrument (JEOL, Japan) from $\mathrm{Pt} / \mathrm{Pd}$ coated samples.

\subsection{Synthesis of polyesters by melt polycondensation}

The homopolyesters were prepared from two-component mixtures with the corresponding compositions: poly(butylene sebacate) (PBSeb) from 1,4-butanediol and diethyl sebacate; poly(glucitylene sebacate), (PGluxSeb) from 2,4:3,5-di-O-methyleneD-glucitol (Glux-diol) and diethyl sebacate (DES); poly(butylene glucarate) (PBGlux) from 1,4-butanediol and dimethyl 2,4:3,5-di-O-methylene-D-glucarate. The copolyesters were prepared from three-component mixtures with varying comonomer ratios: poly(butylene-co-glucitylene sebacate) $\left(\mathrm{PB}_{\mathrm{x}} \mathrm{Glux}_{\mathrm{y}} \mathrm{Seb}\right)$ from 1,4-butanediol, 2,4:3,5-diO-methylene-D-glucitol and diethyl sebacate. In the abbreviated name of these copolyesters $x$ and $y$ subscripts indicate the mol percentages (\%-mol) of butylene and 2,4:3,5-di-O-methylene-D-glucitylene units, respectively. In turn PBSeb Glux $_{y}$ copolyesters were obtained from 1,4-butanediol, diethyl sebacate and dimethyl 2,4:3,5di-O-methylene-D-glucarate. In this case $x$ and $y$ subscripts refer to mol percentages (\%-mol) of sebacate and 2,4:3,5-di-O-methylene-D-glucarate units, respectively.

The reactions were performed in two steps (transesterification and polycondensation) in a three necked cylindrical-bottom flask equipped with a mechanical stirrer, a nitrogen inlet and a vacuum distillation outlet. In both series an excess of diol to diester was used, and dibutyl tin oxide (DBTO) was added as a catalyst $(0.6 \mathrm{~mol} \%$ respect to the diester). The apparatus was vented with nitrogen several times at room temperature in order to remove air and to avoid oxidation during polymerization. The transesterification reaction was carried out under a low nitrogen flow whereas polycondensation was conducted under a 0.03-0.06 mbar vacuum and temperatures for each step were carefully selected for each system. Once the reaction was over, the mixture was cooled to room temperature and the atmospheric pressure 
was recovered with nitrogen to prevent degradation. The resulting polymers were dissolved in chloroform and precipitated in an excess of methanol in order to remove unreacted monomers and formed oligomers. Finally, the polymer was collected by filtration, extensively washed with methanol, and dried under vacuum. These samples coming directly from synthesis without any further treatment were used for characterization. The detailed conditions applied for the synthesis of homopolyesters and copolyesters were the following:

PBSeb homopolyester. BD to DES molar ratio:1.5/1. Transesterification, $180^{\circ} \mathrm{C}, 3 \mathrm{~h}$; polycondensation, $205^{\circ} \mathrm{C}, 2.5 \mathrm{~h}$.

${ }^{1} \mathrm{H}$ NMR (300 MHz, $\mathrm{CDCl}_{3}$ ), $\delta$ (ppm): 4.09 (bt, $-\mathrm{OCH}_{2} \mathrm{CH}_{2}$ ), 2.29 (t, $-\mathrm{OCOCH}_{2}$ ), 1.70 (bt, $-\mathrm{OCH}_{2} \mathrm{CH}_{2}$ ), 1.61 (m, -OCOCH${ }_{2} \mathrm{CH}_{2}$ ), 1.30 (bs, $-\mathrm{OCOCH}_{2} \mathrm{CH}_{2} \mathrm{CH}_{2} \mathrm{CH}_{2}$ ). ${ }^{13} \mathrm{C}\left\{{ }^{1} \mathrm{H}\right\}-\mathrm{NMR}$ $\left(\mathrm{CDCl}_{3}, 75.5 \mathrm{MHz}\right), \delta(\mathrm{ppm}): 173.7(\mathrm{C}=\mathrm{O}), 63.6\left(\mathrm{OCH}_{2}\right), 34.2\left(-\mathrm{OCOCH}_{2}\right) 29.0$ $\left(\mathrm{OCOCH}_{2} \mathrm{CH}_{2} \mathrm{CH}_{2}\right), 25.3\left(-\mathrm{OCH}_{2} \mathrm{CH}_{2}\right), 24.9\left(-\mathrm{OCOCH}_{2} \mathrm{CH}_{2}\right)$.

$P B_{x}$ Glux Seb copolyesters. BD and Glux-diol mixture to DES molar ratio: 1.2/1.

$\mathrm{PB}_{90} \mathrm{Glux}_{10}$ Seb: transesterification, $170^{\circ} \mathrm{C}, 3.5 \mathrm{~h}$; polycondensation, $170{ }^{\circ} \mathrm{C}, 5 \mathrm{~h}$.

$\mathrm{PB}_{80} \mathrm{Glux}_{20}$ Seb: transesterification, $145^{\circ} \mathrm{C}, 7 \mathrm{~h}$; polycondensation, $145^{\circ} \mathrm{C}, 8 \mathrm{~h}$.

$\mathrm{PB}_{71}$ Glux $_{29}$ Seb: transesterification, $140^{\circ} \mathrm{C}, 14 \mathrm{~h}$; polycondensation, $140^{\circ} \mathrm{C}, 10 \mathrm{~h}$.

$\mathrm{PB}_{65} \mathrm{Glux}_{35}$ Seb: transesterification, $135^{\circ} \mathrm{C}, 18 \mathrm{~h}$; polycondensation, $135^{\circ} \mathrm{C}, 15 \mathrm{~h}$.

${ }^{1} \mathrm{H}$ NMR (300 MHz, $\left.\mathrm{CDCl}_{3}\right), \delta$ (ppm): 5.21 (d, $\left.\mathrm{OCH}_{2} \mathrm{O}\right), 5.00$ (s, $\left.\mathrm{OCH}_{2} \mathrm{O}\right), 4.78$ (d, $\mathrm{OCH}_{2} \mathrm{O}$ ), 4.51-4.21 (m, - $\left.\mathrm{OCH}_{2} \mathrm{CH}(\mathrm{Glux})\right), 4.16$ (m, CH), 4.10 (bt, $\left.\mathrm{OCH}_{2}(\mathrm{~B})\right), 3.87$ (bt, $\mathrm{CH}), 3.73$ (bs, CH), 3.56 (bs, CH), $2.29\left(\mathrm{~m},-\mathrm{OCOCH}_{2}\right), 1.70\left(\mathrm{bm},-\mathrm{OCH}_{2} \mathrm{CH}_{2}\right), 1.61$ (m, $\left.-\mathrm{OCOCH}_{2} \mathrm{CH}_{2}\right), 1.30$ (s, $\left.-\mathrm{OCOCH}_{2} \mathrm{CH}_{2} \mathrm{CH}_{2} \mathrm{CH}_{2}\right) \cdot{ }^{13} \mathrm{C}\left\{{ }^{1} \mathrm{H}\right\}-\mathrm{NMR}\left(\mathrm{CDCl}_{3}, 75.5 \mathrm{MHz}\right), \delta$ (ppm): 173.5-173.3 (C=O), $92.9\left(\mathrm{OCH}_{2} \mathrm{O}\right), 88.4\left(\mathrm{OCH}_{2} \mathrm{O}\right), 75.6(\mathrm{CH}), 73.8(\mathrm{CH}), 71.0$ $(\mathrm{CH}), 67,3(\mathrm{CH}), 63.7\left(\mathrm{OCH}_{2}\right), 63.1\left(\mathrm{OCH}_{2}\right), 60.4\left(\mathrm{OCH}_{2}\right), 34.2-34.0\left(-\mathrm{OCOCH}_{2}\right), 29.1$ $\left(\mathrm{CH}_{2}\right), 25.4\left(\mathrm{CH}_{2}\right), 24.9-24.8\left(\mathrm{CH}_{2}\right)$.

PBSeb Glux $_{y}$ copolyesters. BD to DES and Glux-diester mixture: 1.5/1. Similar temperatures were applied in this case for all the compositions, and reaction times 
were increased according to the Glux content. Transesterification, $175^{\circ} \mathrm{C}, 4.5-15 \mathrm{~h}$; polycondensation, $175^{\circ} \mathrm{C}, 10-24 \mathrm{~h}$.

${ }^{1} \mathrm{H}$ NMR (300 MHz, CDCl 3 ), $\delta(\mathrm{ppm}): 5.30\left(\mathrm{~d}, \mathrm{OCH}_{2} \mathrm{O}\right), 5.06-4.99\left(2 \mathrm{dd}, \mathrm{OCH}_{2} \mathrm{O}\right), 4.83$ (d, $\mathrm{OCH}_{2} \mathrm{O}$ ), 4.59 (bs, CH), 4.33 (bs, CH), 4.27 (bt, OCH $(\mathrm{Glux})$ ), 4.09 (m, OCH$(\mathrm{B})$, $\mathrm{CH}), 4.02$ (bs, $\mathrm{CH}), 2.28$ (t, $\left.-\mathrm{OCOCH}_{2}\right), 1,75-1.66\left(\mathrm{~m},-\mathrm{OCH}_{2} \mathrm{CH}_{2}\right), 1.60(\mathrm{~m}-$ $\left.\mathrm{OCOCH}_{2} \mathrm{CH}_{2}\right), 1.29$ (s, -OCOCH $\left.\mathrm{CH}_{2} \mathrm{CH}_{2} \mathrm{CH}_{2}\right) .{ }^{13} \mathrm{C}\left\{{ }^{1} \mathrm{H}\right\}-\mathrm{NMR}\left(\mathrm{CDCl}_{3}, 75.5 \mathrm{MHz}\right), \delta$ (ppm): 173.8-167.1 (C=O), $92.5\left(\mathrm{OCH}_{2} \mathrm{O}\right), 90.1\left(\mathrm{OCH}_{2} \mathrm{O}\right), 76.4(\mathrm{CH}), 75.4(\mathrm{CH}), 70.3$ $(\mathrm{CH}), 68.7(\mathrm{CH})$, 65.5-63.4 $\left(\mathrm{OCH}_{2}\right), 34.2\left(\mathrm{CH}_{2}\right) 29.1\left(\mathrm{CH}_{2}\right)$, 25.3-25.1 $\left(\mathrm{CH}_{2}\right), 24.9\left(\mathrm{CH}_{2}\right)$. PBGlux homopolyester. BD to dimethyl 2,4:3,5-di-O-methylene-D-glucarate molar ratio: $2.2 / 1$. Transesterification, $170{ }^{\circ} \mathrm{C}, 8 \mathrm{~h}$; polycondensation: $170^{\circ} \mathrm{C}, 8 \mathrm{~h}$.

${ }^{1} \mathrm{H}$ NMR ( $\left.\mathrm{CDCl}_{3} / \mathrm{TFA}, 300 \mathrm{MHz}\right), \delta(\mathrm{ppm}): 5.35$ (d, $\left.\mathrm{OCH}_{2} \mathrm{O}\right), 5.20-4.85$ (2dd, $\left.\mathrm{OCH}_{2} \mathrm{O}\right)$, $4.90\left(\mathrm{~d}, \mathrm{OCH}_{2} \mathrm{O}\right), 4.74(\mathrm{~s}, \mathrm{CH}), 4.50-4.70(\mathrm{~m}, \mathrm{CH}), 4.52-4.20\left(\mathrm{~m},-\mathrm{OCH}_{2} \mathrm{CH}_{2}, \mathrm{CH}\right), 4.12$ (s, CH), 1.79 (s, $\left.-\mathrm{OCH}_{2} \mathrm{CH}_{2}\right) \cdot{ }^{13} \mathrm{C}\left\{{ }^{1} \mathrm{H}\right\}-N M R\left(\mathrm{CDCl}_{3} / \mathrm{TFA}, 75.5 \mathrm{MHz}\right), \delta$ (ppm): 172.5$171.3(\mathrm{C}=\mathrm{O}), 92.3\left(\mathrm{OCH}_{2} \mathrm{O}\right), 90.2\left(\mathrm{OCH}_{2} \mathrm{O}\right), 76.2(\mathrm{CH}), 75.0(\mathrm{CH}), 70.3(\mathrm{CH}), 68.7$ $(\mathrm{CH}), 66.9-66.1\left(\mathrm{OCH}_{2} \mathrm{CH}_{2}\right), 24.7\left(\mathrm{OCH}_{2} \mathrm{CH}_{2}\right)$.

\subsection{Synthesis of polyesters by enzymatic polycondensation}

The low solubility of Glux-diol in organic solvents constitutes a severe limitation for the enzymatic polycondensation of mixtures containing this compound. Several high boiling point solvents of common use in enzymatic polymerizations were essayed with the mixtures made of BD/Glux-diol/DES (9:1:10) and BD/DES/Glux-diester (1:9:1) corresponding to the synthesis of copolyesters $\mathrm{PB}_{90} \mathrm{Glux}_{10}$ Seb and PBSeb ${ }_{90} \mathrm{Glux}_{10}$, respectively. Polymerization was found to proceed in DPO whereas no reaction was observed when either dodecane or diglyme (diethylene glycol dimethyl ether) were the selected solvents. An assortment of reaction conditions including reagent and enzyme concentrations, pressure, temperature and time were tried in order to optimize the process. The reaction parameters finally for the two series selected are summarized in Table 1. 
Table 1. Reaction conditions for the enzymatic synthesis of copolyesters.

\begin{tabular}{|c|c|c|c|c|c|}
\hline Copolymer & Solvent $^{a}$ & $\begin{array}{l}\text { [CALB] } \\
\%(w / w)\end{array}$ & $\begin{array}{c}T^{\mathrm{b}} \\
\left({ }^{\circ} \mathrm{C}\right)\end{array}$ & $\begin{array}{c}P^{b} \\
\text { (bars) }\end{array}$ & $\begin{array}{l}t^{\mathrm{b}} \\
(\mathrm{h})\end{array}$ \\
\hline $\mathrm{PB}_{90} \mathrm{Glux}_{10} \mathrm{Seb}$ & DPO & 10 & $90 / 90$ & $1 / 0.04$ & $5 / 72$ \\
\hline PBSeb $_{90}$ Glux $_{10}$ & DPO & 10 & $85 / 85$ & $1 / 0.04$ & $5 / 72$ \\
\hline
\end{tabular}

As an illustrative example, the experimental details used for the synthesis of PBSeb $_{90}$ Glux $_{10}$ are given: $0.2 \mathrm{~g}$ of CALB (10\% w/w of total monomer), $5.78 \mathrm{mmol}$ of DES $(1.345 \mathrm{~g}), 5.78 \mathrm{mmol}$ of Glux-diester $(0.1516 \mathrm{~g}), 5.85 \mathrm{mmol}$ of BD $(0.5277 \mathrm{~g})$ and molecular sieves $(\sim 1.5 \mathrm{~g})$ were mixed with $6 \mathrm{~mL}$ of DPO. The mixture was magnetically stirred and heated at $85{ }^{\circ} \mathrm{C}$ under nitrogen at atmospheric pressure for $5 \mathrm{~h}$ to convert the monomers into oligomers. In the second step, the pressure was reduced to 0.1 bars and the reaction left to proceed for $12 \mathrm{~h}$ followed by a period of $72 \mathrm{~h}$ at 0.04 bars in order to eliminate the ethanol that is released in the transesterification reaction. At the end of reaction the mixture was dissolved in chloroform and then filtered to remove the enzyme and the molecular sieves. The chloroform solution was slowly poured into methanol under stirring to precipitate the copolyester as a white crystalline product. These conditions were then used for the synthesis of other copolyesters of the series.

\subsection{Hydrolytic and enzymatic degradation assays}

Both hydrolytic and enzymatic degradation essays were carried out for a representative selection of polyesters. First, films of polyesters and copolyesters with a thickness of about $200 \mu \mathrm{m}$ were prepared by melt compression followed by quenching into ice-cold water. Then the films were cut into $10 \mathrm{~mm}$-diameter disks and dried under vacuum to constant weight, which finally remained between 20 and $30 \mathrm{mg}$. The disks were individually immersed in vials containing $10 \mathrm{~mL}$ of either sodium citric acid buffer $\mathrm{pH} 2.0$ (hydrolytic degradation), or sodium phosphate buffer $\mathrm{pH} 7.4$ containing $10 \mathrm{mg}$ of porcine pancreas lipases (enzymatic degradation), and left for incubation at $37^{\circ} \mathrm{C}$ for four weeks. The buffered enzyme solution was replaced every $72 \mathrm{~h}$ to maintain the 
enzyme activity. The disks were withdrawn from the incubation medium at scheduled periods of time, washed thoroughly with distilled water, and dried to constant weight.

\section{Results and discussion}

\subsection{Melt polycondensation-synthesis and microstructure}

In this work two series of copolyesters of PBSeb were synthesized using as comonomer either 2,4:3,5-di-O-methylene D-glucitol (Glux-diol) or dimethyl 2,4:3,5-diO-methylene D-glucarate (Glux-diester), both of which derived from D-glucose (Scheme 2). The two series differ therefore in which unit of PBSeb, diol or diacid, was replaced by Glux.

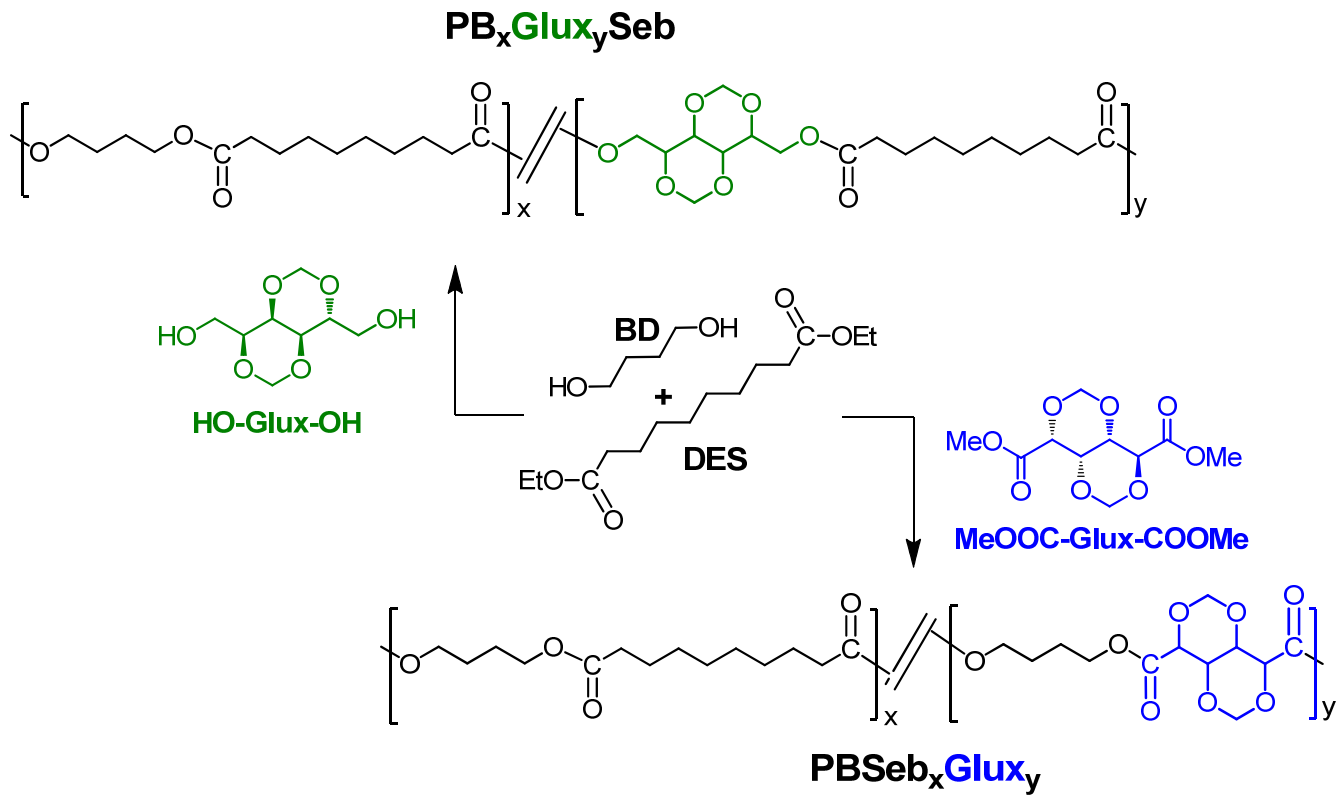

Scheme 2. Polymerization reactions leading to $\mathrm{PB}_{\mathrm{x}} \mathrm{Glux}_{\mathrm{y}} \mathrm{Seb}$ and $\mathrm{PBSeb}_{\mathrm{x}} \mathrm{Glux}_{\mathrm{y}}$. Green and blue colors are used to identify the diol and diacid Glux units, respectively.

The polymerization reactions were carried out in the melt in the total absence of solvents in order to imitate as far as possible the conditions usually applied in industrial practice. In the synthesis of $\mathrm{PB}_{\mathrm{x}} \mathrm{Glux}$ Seb, BD was partially replaced by Gluxdiol, so a series of copolyesters with contents in Glux units ranging from 10 to $35 \%$ were prepared in addition to the homopolyester made entirely of Glux units. In the first step, transesterification of DES leading to $\mathrm{PB}_{\mathrm{x}} \mathrm{Glux} \mathrm{y}$ Seb oligomers was accomplished at 
temperatures between $135-170{ }^{\circ} \mathrm{C}$ under atmospheric pressure with formation of ethanol and methanol, which were removed by distillation. Higher temperatures were used for higher contents of Glux units. Since Glux-diol was not readily soluble in the reaction mixture special attention was paid to get it homogeneous before starting. As transesterification advanced the appearance of the mixture changed from transparent to opaque due to the precipitation of insoluble oligomers containing glucitylene units. Polycondensation of the oligomers leading to $\mathrm{PB}_{\mathrm{x}} \mathrm{Glux}_{\mathrm{y}} \mathrm{Seb}$ copolyesters took place in the second step at $130-170{ }^{\circ} \mathrm{C}$ under vacuum with releasing of excess of diols and the appearance of the mass evolving from opaque to transparent. A similar procedure was applied for the synthesis of PBSeb $\mathrm{Glux}_{\mathrm{y}}$. In this case DES was partially replaced by Glux-diester to prepare a series of copolyesters with contents in Glux units up to $50 \%$.

For comparison purposes, the homopolyesters PBSeb and PBGlux were also prepared using essentially the same polycondensation procedure as for copolyesters with some minor modifications according to the case. Unfortunately the synthesis of PGluxSeb was not feasible by melt polymerization because Glux-diol remained insolubilized in the reaction mixture even at high temperatures. As it will be discussed below this homopolyester could be however obtained by polycondensation in solution mediated by CALB.

Synthesis results obtained for the two copolyester series together with those for the parent homopolyesters are compared in Table 1. All copolyesters were prepared in yields around $80-90 \%$ after purification and they have intrinsic viscosities ranging from 0.50 to $0.90 \mathrm{dL} \cdot \mathrm{g}^{-1}$ and weight-average molecular weights roughly between 25,000 and $40,000 \mathrm{~g} \cdot \mathrm{mol}^{-1}$ with dispersities within the 2.2-2.4 range. The chemical constitution of the polyesters was ascertained by both ${ }^{1} \mathrm{H}$ and ${ }^{13} \mathrm{C}$ NMR spectroscopy. Illustrative ${ }^{1} \mathrm{H}$ and ${ }^{13} \mathrm{C}$ spectra of representative members of each series as well as the $2 \mathrm{D} N M R$ spectra (cosy and hetcor) used for the assignment of the peaks are available in the Supplementary Electronic Information (ESI) document. The comonomeric composition of $\mathrm{PB}_{\mathrm{x}} \mathrm{Glux}$ Seb and $\mathrm{PBSeb}_{\mathrm{x}} \mathrm{Glux}_{\mathrm{y}}$ copolyesters was determined by comparing the 
integrated signals arising from protons contained exclusively in each of their two respective repeating units (butylene and glucitylene protons for the former and sebacate and glucarate protons for the latter). 
Table 1. Composition and molecular weight of $\mathrm{PB}_{\mathrm{x}} \mathrm{Glux} \mathrm{x}_{\mathrm{Seb}}$ and $\mathrm{PBSeb} \mathrm{Glux}_{\mathrm{y}}$ prepared by melt polycondensation.

\begin{tabular}{|c|c|c|c|c|c|c|c|c|c|c|c|c|c|}
\hline \multirow{4}{*}{ Copolyester } & \multirow{4}{*}{$\begin{array}{c}\text { Yield } \\
(\%)\end{array}$} & \multicolumn{8}{|c|}{ Composition $^{a}$} & \multirow{3}{*}{\multicolumn{4}{|c|}{ Molecular weight }} \\
\hline & & \multicolumn{4}{|c|}{ Feed } & \multicolumn{4}{|c|}{ Copolyester } & & & & \\
\hline & & \multicolumn{2}{|c|}{ Diol } & \multicolumn{2}{|c|}{ Diacid } & \multicolumn{2}{|c|}{ Diol } & \multicolumn{2}{|c|}{ Diacid } & & & & \\
\hline & & {$[\mathrm{B}]$} & [Glux] & [Seb] & [Glux] & {$[\mathrm{B}]$} & [Glux] & [Seb] & [Glux] & {$[\eta]^{\mathrm{b}}$} & $M_{\mathrm{n}}{ }^{\mathrm{c}}$ & $M_{\mathrm{w}}{ }^{\mathrm{c}}$ & $\oplus^{c}$ \\
\hline PBSeb & 93 & 100 & 0 & 100 & 0 & 100 & 0 & 100 & 0 & 0.90 & 19,800 & 43,500 & 2.2 \\
\hline \multicolumn{14}{|l|}{ PBxGluxyseb } \\
\hline $\mathrm{PB}_{90} \mathrm{Glux}_{10} \mathrm{Seb}$ & 89 & 90 & 10 & 100 & 0 & 90 & 10 & - & - & 0.87 & 17,900 & 41,100 & 2.3 \\
\hline $\mathrm{PB}_{80} \mathrm{Glux}_{20} \mathrm{Seb}$ & 87 & 80 & 20 & 100 & 0 & 80 & 20 & - & - & 0.79 & 17,100 & 37,700 & 2.2 \\
\hline $\mathrm{PB}_{71} \mathrm{Glux}_{29} \mathrm{Seb}$ & 85 & 70 & 30 & 100 & 0 & 71 & 29 & - & - & 0.86 & 17,800 & 41,000 & 2.3 \\
\hline $\mathrm{PB}_{65} \mathrm{Glux}_{35} \mathrm{Seb}$ & 80 & 60 & 40 & 100 & 0 & 65 & 35 & - & - & 0.53 & 10,400 & 25,000 & 2.4 \\
\hline PGluxSeb* & 70 & 0 & 100 & 100 & 0 & 0 & 100 & - & - & 0.40 & 5,600 & 11,200 & 2.0 \\
\hline \multicolumn{14}{|l|}{ PBSebx Glux $_{y}$} \\
\hline PBSeb $_{91}$ Glux $_{9}$ & 87 & 100 & 0 & 90 & 10 & - & - & 91 & 9 & 0.70 & 14,300 & 33,000 & 2.3 \\
\hline PBSeb $_{80}$ Glux $_{20}$ & 86 & 100 & 0 & 80 & 20 & - & - & 80 & 20 & 0.74 & 15,800 & 34,900 & 2.2 \\
\hline $\mathrm{PBSeb}_{72} \mathrm{Glux}_{28}$ & 86 & 100 & 0 & 70 & 30 & - & - & 72 & 28 & 0.70 & 14,300 & 33,000 & 2.3 \\
\hline PBSeb $_{60}$ Glux $_{40}$ & 83 & 100 & 0 & 60 & 40 & - & - & 60 & 40 & 0.50 & 10,200 & 23,400 & 2.3 \\
\hline PBSeb $_{50}$ Glux $_{50}$ & 80 & 100 & 0 & 50 & 50 & - & - & 50 & 50 & 0.67 & 13,300 & 31,900 & 2.4 \\
\hline PBGlux & 50 & 100 & 0 & 0 & 100 & - & - & 0 & 100 & 0.46 & 6,900 & 16,500 & 2.4 \\
\hline
\end{tabular}

${ }^{\mathrm{a}}$ Molar composition in the feed and in the copolyester as determined by integration of ${ }^{1} \mathrm{H}$ NMR spectra.

Intrinsic viscosity in $\mathrm{dL} \cdot \mathrm{g}^{-1}$ measured in DCA at $25^{\circ} \mathrm{C}$

${ }^{\mathrm{C}}$ Number and weight average molecular weights in $\mathrm{g} \cdot \mathrm{mol}^{-1}$, and dispersities measured by GPC in HFIP against PMMA standards.

*Polymer synthesized by enzymatic polymerization. 
The percentage of sugar units present in the copolyesters was estimated by applying simple proportion calculations. It was found that the composition of PBSeb $_{x} G_{l u x}$ copolyesters was essentially the same as that of their respective feeds whereas lower contents of Glux units were incorporated in $\mathrm{PB}_{\mathrm{x}} \mathrm{Glux}_{\mathrm{y}} \mathrm{Seb}$ copolyesters, this probably due to the poor miscibility of the monomers in the reaction media.

The microstructure of the PBSeb Glux $_{y}$ series was analyzed by NMR. The ${ }^{13} \mathrm{C}$ NMR spectra of these copolyesters showed complex signals for the butylene units revealing that the methylene carbons are sensitive to sequence effects. As it is shown in Figure 1, nine peaks, two of them overlapped, appear within 65.6-63.3 ppm interval of the spectra, which correspond to the four types of dyads (SebSeb, GluxSeb/SebGlux, GluxGlux) that are possible along the copolyester chain. The SebSeb dyad shows a peak for the first methylene of the butylene unit at $63.7 \mathrm{ppm}$ (signal 1).Due to the lack of symmetry of the Glux structure, GluxSeb or SebGlux dyads display four peaks instead of two for this methylene at $63.4,63.5,65.2$ and 65.5 ppm (signals 2 and 3). The same occurs with GluxGlux dyad; this dyad displays four peaks, instead of one, at 64.9 and $65.0 \mathrm{ppm}$ in addition to two peaks overlapped with the signal at $65.2 \mathrm{ppm}$ of the GluxSeb/SebGlux dyads (signals 4). The peaks assignment was carried out by comparison with the ${ }^{13} \mathrm{C}$ NMR spectra recorded from PBSeb and PBGlux homopolyesters. By Lorentzian deconvolution of the peaks using the WinNMR software, the number-average lengths $n$ of butylene-sebacate (BS) and butylene-glucarate $(\mathrm{BG})$ sequences as well as the degree of randomness $(R)$ could be calculated. The equations used for these calculations were the following,

$$
\begin{gathered}
n_{\mathrm{BS}}=\left(\mathrm{N}_{\mathrm{SS}}+1 / 2\left(\mathrm{~N}_{\mathrm{SG}}+\mathrm{N}_{\mathrm{GS}}\right)\right) / 1 / 2\left(\mathrm{~N}_{\mathrm{SG}}+\mathrm{N}_{\mathrm{GS}}\right) \\
n_{\mathrm{BG}}=\left(\mathrm{N}_{\mathrm{GG}}+1 / 2\left(\mathrm{~N}_{\mathrm{SG}}+\mathrm{N}_{\mathrm{GS}}\right)\right) / 1 / 2\left(\mathrm{~N}_{\mathrm{SG}}+\mathrm{N}_{\mathrm{GS}}\right) \\
R=\left(1 / n_{\mathrm{BS}}\right)+\left(1 / n_{\mathrm{BG}}\right)
\end{gathered}
$$

and results are given in Table 2 showing that the microstructure of PBSeb Glux $_{y}$ copolyesters was random for every composition with $R$ values very close to unity. 
Unfortunately the microstructure of the $\mathrm{PB}_{\mathrm{x}} \mathrm{Glux}_{\mathrm{y}} \mathrm{Seb}$ series could not be determined because the octamethylene segment of the sebacate unit attenuates the environmental mutual electronic effect of butylene and glucitylene units to the point that no differences due to sequence distribution could be detected in the ${ }^{13} \mathrm{C}$ spectra of these copolyesters. Nevertheless, taking into account preceding data on the microstructure of other Glux containing copolyesters, ${ }^{22}$ as well as the DSC behavior of this series which will be described below, it can be reasonably assumed that a random sequence distribution of butylene and glucitylene units must also occur in $\mathrm{PB}_{\mathrm{x}} \mathrm{Glux}$ Seb copolyesters.
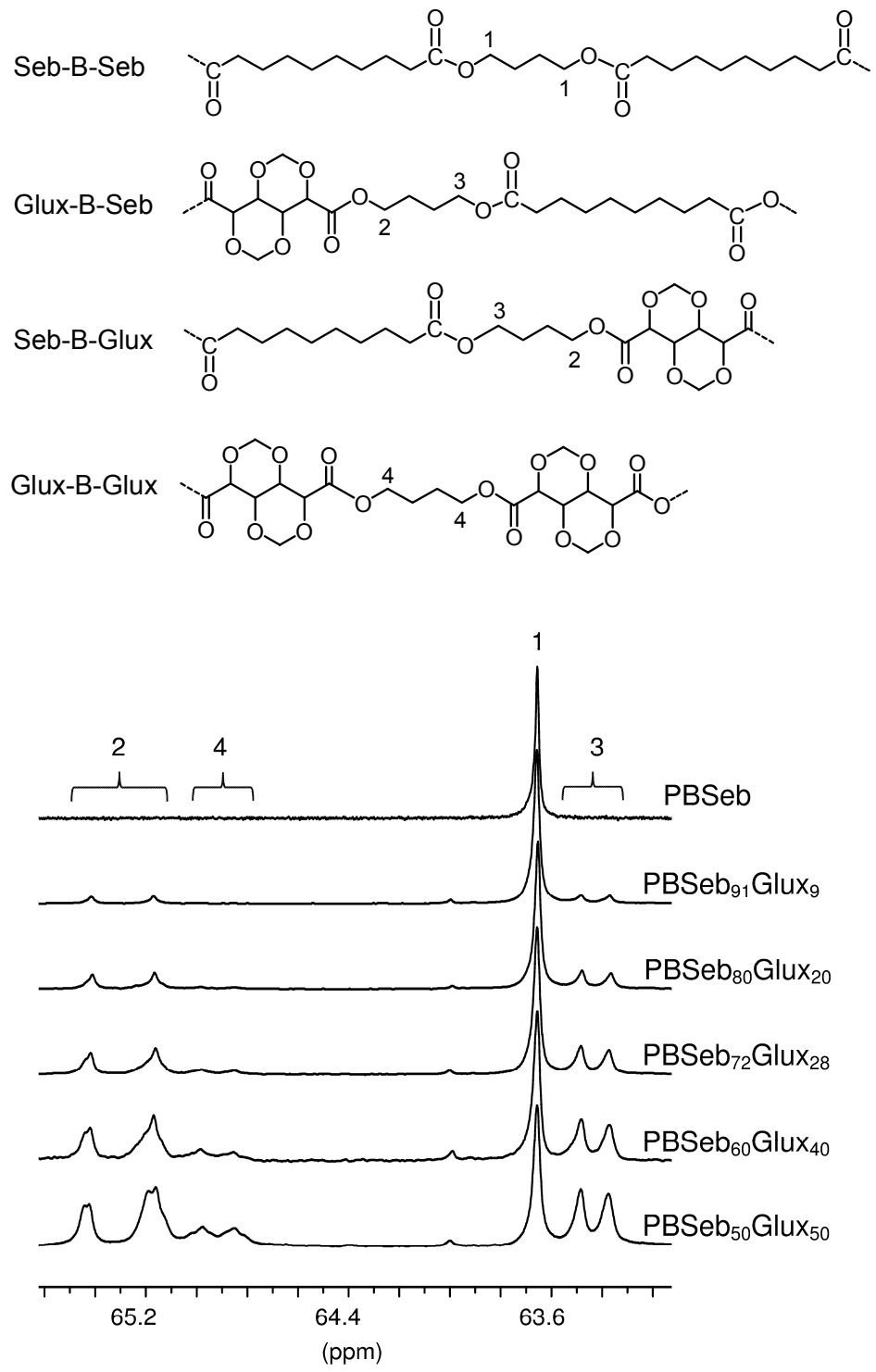

Figure 1. The four possible dyads present in $\mathrm{PBSeb}_{\mathrm{x}} \mathrm{Glux}_{\mathrm{y}}$ copolyesters (top); ${ }^{13} \mathrm{C}$ NMR spectra in the resonance region of the first oxybutylene carbon for PBSeb homopolyester and PBSeb Glux $_{y}$ copolyesters (bottom). 
Table 2. Composition and microstructure of PBSeb $\mathrm{Glux}_{\mathrm{y}}$ copolyesters.

\begin{tabular}{|c|c|c|c|c|c|c|c|c|}
\hline \multirow[t]{2}{*}{ Copolyester } & \multicolumn{2}{|c|}{ Composition $^{a}$} & \multicolumn{3}{|c|}{$\begin{array}{l}\text { Dyad content } \\
(\%)^{\mathrm{b}}\end{array}$} & \multicolumn{2}{|c|}{$\begin{array}{c}\text { Number } \\
\text { average } \\
\text { sequence } \\
\text { lengths }{ }^{c}\end{array}$} & \multirow[t]{2}{*}{$R^{\mathrm{d}}$} \\
\hline & $X_{\text {Seb }}$ & $X_{\text {Glux }}$ & SS & SG/GS & GG & $n_{\mathrm{BS}}$ & $n_{\mathrm{BG}}$ & \\
\hline PBSeb $_{91}$ Glux $_{9}$ & 0.91 & 0.09 & $\begin{array}{c}81.3 \\
(82.8)\end{array}$ & $\begin{array}{c}18.7 \\
(16.4)\end{array}$ & $\begin{array}{l}0.0 \\
(0.8)\end{array}$ & $\begin{array}{c}9.7 \\
(11.2)\end{array}$ & $\begin{array}{c}1.0 \\
(1.1)\end{array}$ & $\begin{array}{l}1.01 \\
(1.0)\end{array}$ \\
\hline PBSeb $_{80}$ Glux $_{20}$ & 0.80 & 0.20 & $\begin{array}{c}63.3 \\
(64.0)\end{array}$ & $\begin{array}{c}32.5 \\
(32.0)\end{array}$ & $\begin{array}{l}4.2 \\
(4.00)\end{array}$ & $\begin{array}{c}4.9 \\
(5.0)\end{array}$ & $\begin{array}{c}1.3 \\
(1.3)\end{array}$ & $\begin{array}{l}0.99 \\
(1.0)\end{array}$ \\
\hline $\mathrm{PBSeb}_{72} \mathrm{Glux}_{28}$ & 0.72 & 0.28 & $\begin{array}{c}49.1 \\
(51.8)\end{array}$ & $\begin{array}{r}42.3 \\
(40.4)\end{array}$ & $\begin{array}{c}8.6 \\
(7.8)\end{array}$ & $\begin{array}{c}3.3 \\
(3.57)\end{array}$ & $\begin{array}{c}1.4 \\
(1.39)\end{array}$ & $\begin{array}{l}1.01 \\
(1.0)\end{array}$ \\
\hline PBSeb $_{60}$ Glux $_{40}$ & 0.60 & 0.40 & $\begin{array}{c}34.0 \\
(36.0)\end{array}$ & $\begin{array}{c}48.5 \\
(48.0)\end{array}$ & $\begin{array}{c}17.5 \\
(16.0)\end{array}$ & $\begin{array}{c}2.4 \\
(2.5)\end{array}$ & $\begin{array}{c}1.7 \\
(1.7)\end{array}$ & $\begin{array}{r}1.0 \\
(1.0)\end{array}$ \\
\hline PBSeb $_{50}$ Glux $_{50}$ & 0.50 & 0.50 & $\begin{array}{l}24.8 \\
(25.0)\end{array}$ & $\begin{array}{c}49.7 \\
(50.0)\end{array}$ & $\begin{array}{c}25.5 \\
(25.0)\end{array}$ & $\begin{array}{c}2.0 \\
(2.0)\end{array}$ & $\begin{array}{c}2.0 \\
(2.0)\end{array}$ & $\begin{array}{l}1.00 \\
(1.0)\end{array}$ \\
\hline
\end{tabular}

${ }^{\mathrm{a}}$ Calculated from the ${ }^{1} \mathrm{H}$ NMR spectra.

${ }^{\mathrm{b}}$ Experimental values obtained by deconvolution of ${ }^{13} \mathrm{C}$ NMR peaks corresponding to the first oxybutylene carbon. Theoretical values (in parentheses) were calculated on the basis of a statistical distribution using the copolyester composition data given in this Table.

Dyads are abbreviated as follows:SS, Seb-B-Seb; SG, Seb-B-Glux; GS, Glux-B-Seb; GG, Glux-B-Glux.

'Number average lengths $n$ of butylene-sebacate (BS) and butylene-glucarate (BG) sequences determined

by equations mentioned in the text.

${ }^{\mathrm{d}} R$ : Degree of randomness.

\subsection{Enzymatic synthesis by CALB}

Given the bio-based character of the system under study and the vulnerability of Glux monomers to high temperatures, the synthesis of the polyesters and copolyesters described above was attempted by enzymatic polycondensation in solution catalyzed by CALB. From previous exploratory experiments it was concluded that DPO was the most suitable solvent for these polymerizations because the mixture became homogeneous as polycondensation proceeded. The NMR analysis of samples taken from the reaction mixture at regular time intervals revealed no changes in the signals arising from DES after $2 \mathrm{~h}$ indicating that the reaction was very slow. It was also noticed that raising the amount of either solvent or enzyme did not affect the reaction rate but made the recovery of the polymer more difficult instead. A detailed account of 
the essays carried out with indication of the applied conditions and results obtained therefrom is provided in the ESI file.

The optimized results achieved in the synthesis of the two series of copolyesters containing Glux units are presented in Table 3. All copolyesters were prepared in yields ranging from 30 to $70 \%$ after purification, with intrinsic viscosities oscillating between 0.3 and $0.44 \mathrm{dL} \cdot \mathrm{g}^{-1}$. Weight-average molecular weights in the proximities of $10,000 \mathrm{~g} \cdot \mathrm{mol}^{-1}$ with dispersities around 2 were measured for all the copolyesters. It is worthy to note that lower molecular weights and yields were attained for PBSeb Glux $_{y}$ than for $\mathrm{PB}_{\mathrm{x}}$ Glux $_{\mathrm{y}}$ Seb copolyesters and that the two parameters decreased monotonically with the Glux content in both series. In line with such results, the PBGlux homopolyester could not be synthesized by this method. A sound explanation for the lower reactivity observed for the Glux-diester is that the proximity of the bulky bicyclic structure to the carboxylate function hinders the access of this group to the active center of the enzyme. Blank experiments involving the same monomer mixtures under the same reaction conditions but in the absence of enzyme did not show signs of transesterification.

The composition of the copolyesters obtained with the concourse of CALB was estimated by NMR by using the same approach described in the previous section for the analysis of the copolyesters prepared by melt polycondensation. It was found that in both series the content of the copolyesters in Glux units was lower than in their respective feeds, which is consistent with the relative lower reactivity expected for the carboxylate and hydroxymethyl functions placed neighboring to the bulky bicyclic groups. The microstructure of the enzymatically produced PBSeb $\mathrm{Glux}_{\mathrm{y}}$ copolyesters was determined by ${ }^{13} \mathrm{C}$ NMR in the same way as for the copolyesters made in the melt, and the number average sequence lengths and randomness degrees thus obtained are given in the ESI document. $R$ values very close to unity were found for all the examined compositions indicating that as it happens in copolyesters obtained by melt 
polycondensation, the comonomer distribution in PBSeb $\mathrm{Glux}_{\mathrm{y}}$ copolyesters obtained from enzymatic polymerization is essentially random.

Due to the reasons given above, the microstructure of $\mathrm{PB}_{\mathrm{x}} \mathrm{Glux}_{\mathrm{y}} \mathrm{Seb}$ series could not be determined. However a close comparison of the ${ }^{13} \mathrm{C}$ NMR spectra registered from $\mathrm{PB}_{\mathrm{x}} \mathrm{Glux}$ Seb copolyesters with similar compositions obtained by either chemical or enzymatic reaction (Figure 2) are practically undistinguishable. It can be inferred therefore that both series must share the same chain microstructure, which is most likely at random.

Table 3. Composition and molecular weight of $\mathrm{PB}_{\mathrm{x}} \mathrm{Glux}_{\mathrm{y}} \mathrm{Seb}$ and $\mathrm{PBSeb}_{\mathrm{x}} \mathrm{Glux}_{\mathrm{y}}$ prepared by enzymatic polycondensation.

\begin{tabular}{|c|c|c|c|c|c|c|c|c|c|c|c|c|c|}
\hline \multirow{4}{*}{ Copolyester } & \multirow{4}{*}{$\begin{array}{c}\text { Yield } \\
(\%)\end{array}$} & \multicolumn{8}{|c|}{ Composition $^{a}$} & \multirow{3}{*}{\multicolumn{4}{|c|}{ Molecular weight }} \\
\hline & & \multicolumn{4}{|c|}{ Feed } & \multicolumn{4}{|c|}{ Copolyester } & & & & \\
\hline & & \multicolumn{2}{|c|}{ Diol } & \multicolumn{2}{|c|}{ Diacid } & \multicolumn{2}{|c|}{ Diol } & \multicolumn{2}{|c|}{ Diacid } & & & & \\
\hline & & {$[B]$} & [Glux] & [Seb] & [Glux] & {$[\mathrm{B}]$} & [Glux] & [Seb] & [Glux] & {$[\eta]^{\mathrm{b}}$} & $M_{n}{ }^{c}$ & $M_{\mathrm{w}}{ }^{\mathrm{c}}$ & $\Xi^{c}$ \\
\hline PBSeb & 80 & 100 & 0 & 100 & 0 & 100 & 0 & 100 & 0 & 0.53 & 6,900 & 13,100 & 1.9 \\
\hline \multicolumn{14}{|l|}{$\mathrm{PB}_{\mathrm{x}}$ Gluxy Seb } \\
\hline $\mathrm{PB}_{92} \mathrm{Glux}_{8} \mathrm{Seb}$ & 73 & 90 & 10 & 100 & 0 & 90 & 10 & - & - & 0.44 & 5,600 & 12,400 & 2.2 \\
\hline $\mathrm{PB}_{87} \mathrm{Glux}_{13} \mathrm{Seb}$ & 74 & 80 & 20 & 100 & 0 & 87 & 13 & - & - & 0.41 & 5,500 & 11,500 & 2.1 \\
\hline $\mathrm{PB}_{78} \mathrm{Glux}_{22} \mathrm{Seb}$ & 72 & 70 & 30 & 100 & 0 & 78 & 22 & - & - & 0.43 & 5,900 & 12,400 & 2.1 \\
\hline $\mathrm{PB}_{74} \mathrm{Glux}_{26} \mathrm{Seb}$ & 71 & 60 & 40 & 100 & 0 & 74 & 26 & - & - & 0.40 & 5,400 & 11,200 & 2.2 \\
\hline $\mathrm{PB}_{70} \mathrm{Glux}_{30} \mathrm{Seb}$ & 71 & 50 & 50 & 100 & 0 & 70 & 30 & - & - & 0.38 & 4,800 & 10,500 & 2.2 \\
\hline PGluxSeb & 70 & 0 & 100 & 100 & 0 & 0 & 100 & - & - & 0.40 & 5,600 & 11,200 & 2.0 \\
\hline \multicolumn{14}{|l|}{ PBSebxGlux } \\
\hline $\mathrm{PBSeb}_{93} \mathrm{Glux}_{7}$ & 70 & 100 & 0 & 90 & 10 & - & - & 91 & 9 & 0.42 & 5,600 & 11,700 & 2.1 \\
\hline PBSeb $_{85}$ Glux $_{15}$ & 68 & 100 & 0 & 80 & 20 & - & - & 85 & 15 & 0.39 & 5,300 & 11,700 & 2.2 \\
\hline PBSeb $_{73}$ Glux $_{27}$ & 60 & 100 & 0 & 70 & 30 & - & - & 73 & 27 & 0.38 & 5,000 & 10,600 & 2.1 \\
\hline PBSeb $_{66}$ Glux $_{34}$ & 40 & 100 & 0 & 60 & 40 & - & - & 66 & 34 & 0.37 & 4,700 & 10,400 & 2.2 \\
\hline PBSeb $_{55}$ Glux $_{45}$ & 35 & 100 & 0 & 50 & 50 & - & - & 55 & 45 & 0.30 & 4,000 & 8,800 & 2.2 \\
\hline
\end{tabular}

${ }^{a}$ Molar composition of the feed and in the copolyester as determined by integration of ${ }^{1} \mathrm{H}$ NMR spectra.

${ }^{\mathrm{b}}$ Intrinsic viscosity in $\mathrm{dL} \cdot \mathrm{g}^{-1}$ measured in DCA at $25^{\circ} \mathrm{C}$.

${ }^{c}$ Number and weight average molecular weights in $\mathrm{g} \cdot \mathrm{mol}^{-1}$, and dispersities measured by GPC in HFIP against PMMA standards. 


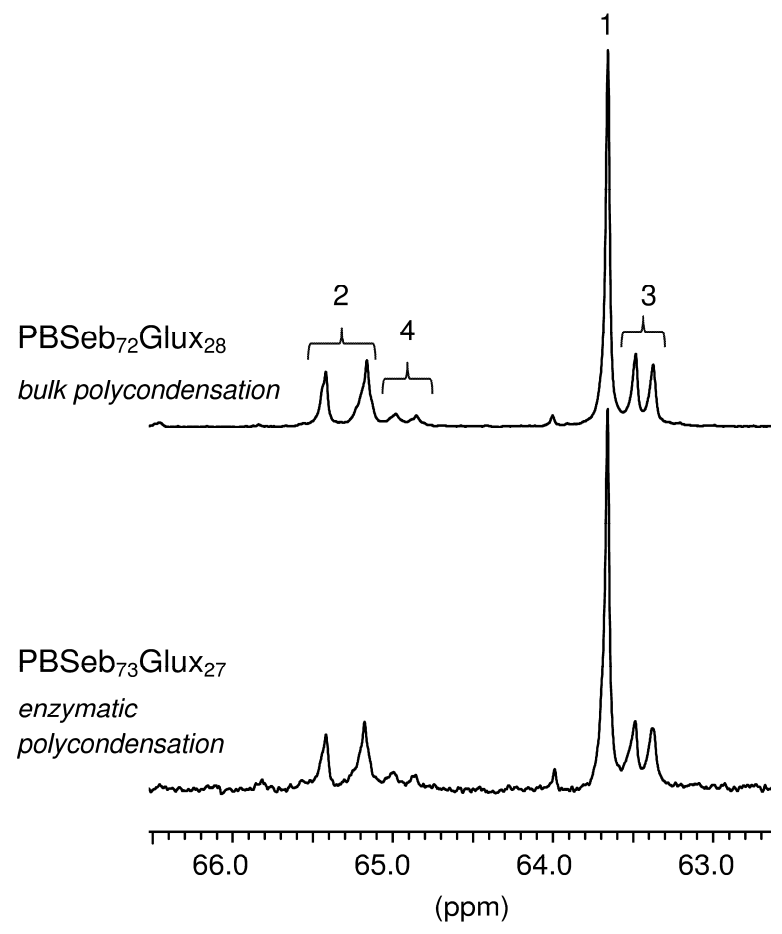

Figure 2. ${ }^{13} \mathrm{C}$ NMR spectra in the region of the first BD methylenes for two PBSeb Glux $_{\mathrm{y}}$ copolyesters prepared by melt polycondensation and enzymatic polymerization, respectively with similar compositions.

\subsection{Thermal properties and X-ray diffraction}

The thermal behaviour of $\mathrm{PB}_{\mathrm{x}} \mathrm{Glux}_{\mathrm{y}}$ Seb and PBSeb $\mathrm{Glux}_{\mathrm{y}}$ copolyesters prepared by melt polycondensation has been examined by TGA and DSC. The thermal properties values resulting from this study are compared in Table 4 where similar data for the parent homopolyesters PBSeb, PGluxSeb and PBGlux are also included for reference. The TGA traces recorded from the whole set of both copolyesters series are comparatively depicted in Figure 3 as well as the derivative curves of the three homopolyesters. In agreement with previously reported data, ${ }^{27}$ PBSeb was observed to decompose in a single stage with the maximum rate taking place at $421{ }^{\circ} \mathrm{C}\left({ }^{\max } T_{\mathrm{d}}\right)$. Noticeably the homopolyester PGluxSeb, which is entirely made of glucitylene units showed an even higher thermal stability with a ${ }^{\max } T_{d}$ close to $432{ }^{\circ} \mathrm{C}$. Such a difference is very probably underestimated since the molecular weight of PGluxSeb is near to fourfold smaller than that of PBSeb. In agreement with these data, decomposition of $\mathrm{PB}_{\mathrm{x}} \mathrm{Glux}_{\mathrm{y}}$ Seb copolyesters was found to take place through a single-step process at 
Table 4. Thermal properties of PBSeb, PGluxSeb, PBGlux, and the PBxGlux Seb, PBSeb $_{x}$ Gluxycopolyesters prepared by melt polycondensation.

\begin{tabular}{|c|c|c|c|c|c|c|c|c|c|c|c|c|}
\hline \multirow{3}{*}{ Polyester } & \multirow{2}{*}{\multicolumn{3}{|c|}{ TGA }} & \multicolumn{9}{|c|}{ DSC } \\
\hline & & & & \multirow[b]{2}{*}{$\begin{array}{c}T_{\mathrm{g}}^{\mathrm{d}} \\
\left({ }^{\circ} \mathrm{C}\right)\end{array}$} & \multicolumn{2}{|c|}{ First Heating $^{e}$} & \multicolumn{2}{|c|}{ Cooling $^{e}$} & \multicolumn{4}{|c|}{ Second Heating ${ }^{e}$} \\
\hline & $\begin{array}{c}T_{\mathrm{d}}{ }^{\mathrm{a}} \\
\left({ }^{\circ} \mathrm{C}\right)\end{array}$ & $\begin{array}{l}T_{\mathrm{ds}}^{\mathrm{b}} \\
\left({ }^{\circ} \mathrm{C}\right)\end{array}$ & $\begin{array}{l}\mathrm{RW}^{\mathrm{c}} \\
(\%)\end{array}$ & & $\begin{array}{c}T_{\mathrm{m}} \\
\left({ }^{\circ} \mathrm{C}\right)\end{array}$ & $\begin{array}{c}\Delta H_{\mathrm{m}} \\
\left(\mathrm{J} \cdot \mathrm{g}^{-1}\right)\end{array}$ & $\begin{array}{c}T_{\mathrm{c}} \\
\left({ }^{\circ} \mathrm{C}\right)\end{array}$ & $\begin{array}{c}\Delta H_{\mathrm{C}} \\
\left(\mathrm{J} \cdot \mathrm{g}^{-1}\right)\end{array}$ & $\begin{array}{c}T_{\mathrm{cc}} \\
\left({ }^{\circ} \mathrm{C}\right)\end{array}$ & $\begin{array}{l}\Delta H_{\mathrm{cc}} \\
\left(\mathrm{J} \cdot \mathrm{g}^{-1}\right)\end{array}$ & $\begin{array}{c}T_{\mathrm{m}} \\
\left({ }^{\circ} \mathrm{C}\right)\end{array}$ & $\begin{array}{c}\Delta H_{m} \\
\left(\mathrm{~J} \cdot \mathrm{g}^{-1}\right)\end{array}$ \\
\hline PBSeb & 395 & 421 & 3 & $-62^{* * *}$ & 65 & 101 & 50 & 82 & - & - & 65 & 88 \\
\hline \multicolumn{13}{|l|}{$\mathrm{PB}_{\mathrm{x}}$ Gluxyseb } \\
\hline PB $_{90} G_{l u x_{10}}$ Seb & 393 & 420 & 5 & n.o. & 56 & 85 & 39 & 63 & - & - & 56 & 67 \\
\hline $\mathrm{PB}_{80} \mathrm{Glux}_{20} \mathrm{Seb}$ & 393 & 419 & 5 & $-50^{\star *}$ & 49 & 64 & 23 & 46 & - & - & 45 & 49 \\
\hline $\mathrm{PB}_{71}$ Glux $_{29}$ Seb & 397 & 423 & 5 & $-14^{*} /-36^{* *}$ & 42 & 42 & - & - & -3 & 12 & 34 & 32 \\
\hline $\mathrm{PB}_{65} \mathrm{Glux}_{35} \mathrm{Seb}$ & 401 & 427 & 2 & $-35^{\star} /-35^{\star *}$ & 40 & 36 & -9 & 10 & 1 & 11 & 33 & 28 \\
\hline *PGluxSeb & 398 & 432 & 6 & $+28^{*}$ & 119 & 32 & 80 & 33 & - & - & 118 & 30 \\
\hline \multicolumn{13}{|l|}{ PBSeb ${ }_{x} G_{l u x}$} \\
\hline PBSeb $_{91}$ Glux $_{9}$ & 381 & $372 / 418$ & 3 & n.o. & 56 & 81 & 34 & 62 & - & - & 54 & 62 \\
\hline PBSeb $_{80}$ Glux $_{20}$ & 361 & $367 / 417$ & 3 & $-49^{* *}$ & 50 & 61 & 21 & 43 & - & - & 48 & 43 \\
\hline $\mathrm{PBSeb}_{72} \mathrm{Glux}_{28}$ & 356 & $367 / 419$ & 6 & $-31^{*} /-42^{* *}$ & 43 & 45 & - & - & 4 & 20 & 35 & 26 \\
\hline PBSeb $_{60}$ Glux $_{40}$ & 364 & $385 / 419$ & 3 & $-25^{\star} /-17^{\star *}$ & 43 & 17 & - & - & - & - & - & - \\
\hline PBSeb $_{50}$ Glux $_{50}$ & 330 & $348 / 417$ & 13 & $-4^{*} /-5^{* *}$ & - & - & - & - & - & - & - & - \\
\hline PBGlux & 274 & 345 & 11 & $+77^{*}$ & - & - & - & - & - & - & - & - \\
\hline
\end{tabular}

${ }^{\mathrm{a}}$ Temperature at which a $10 \%$ weight loss was observed in the TGA traces recorded at $10{ }^{\circ} \mathrm{C} \cdot \mathrm{min}^{-1}$. ${ }^{\mathrm{b}} \mathrm{Temperature}$ of maximum degradation rate. ${ }^{\mathrm{c}}$ Remaining weight at the $600{ }^{\circ} \mathrm{C}$. ${ }^{\mathrm{d}}$ Glass-transition temperature: n.o., not observed; ${ }^{*}$ Data obtained at a constant heating rate of $20{ }^{\circ} \mathrm{C} \cdot \mathrm{min}{ }^{-1}$; ${ }^{* *} \mathrm{Data}$ obtained by DDSC. ${ }^{* *}$ Data taken from literature. ${ }^{[28]}$ e Melting $\left(T_{\mathrm{m}}\right)$ and crystallization $\left(T_{\mathrm{c}}\right)$ temperatures and their respective enthalpies $\left(\Delta H_{m}, \Delta H_{c}\right)$ measured by DSC at heating/cooling rates of $10^{\circ} \mathrm{C} \cdot \mathrm{min}^{-1} ; \mathrm{T}_{\mathrm{cc}}, \Delta H_{\mathrm{cc}}$-cold crystallization. 
a)

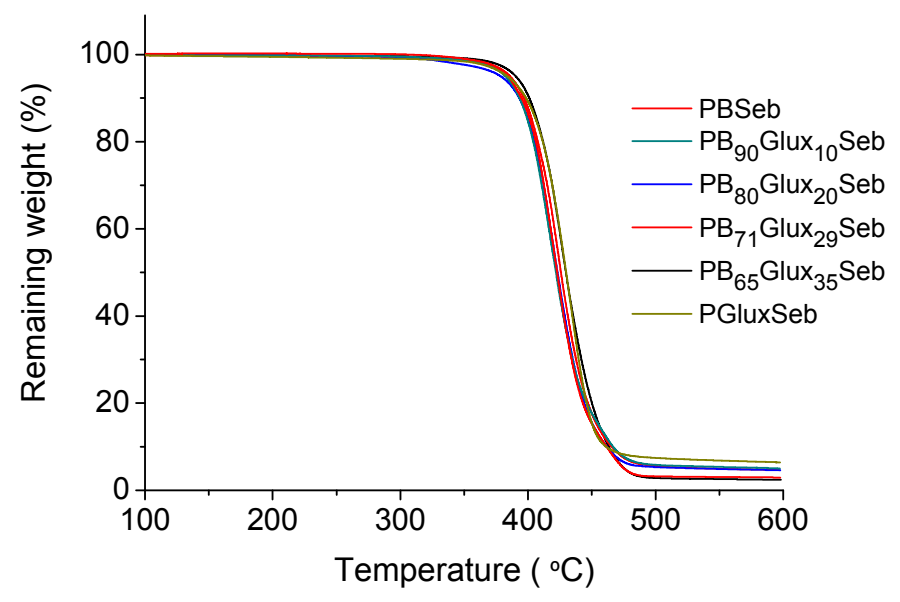

b)

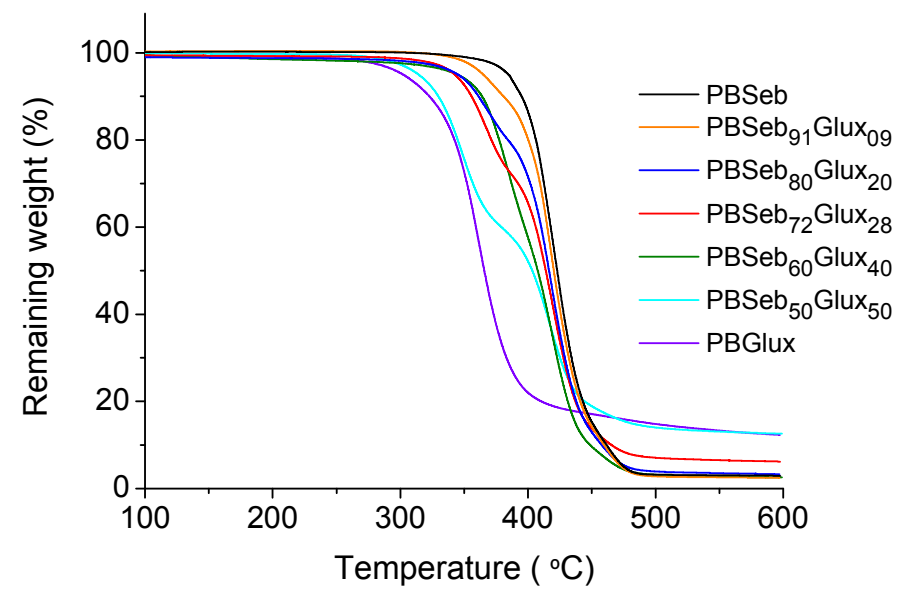

c)

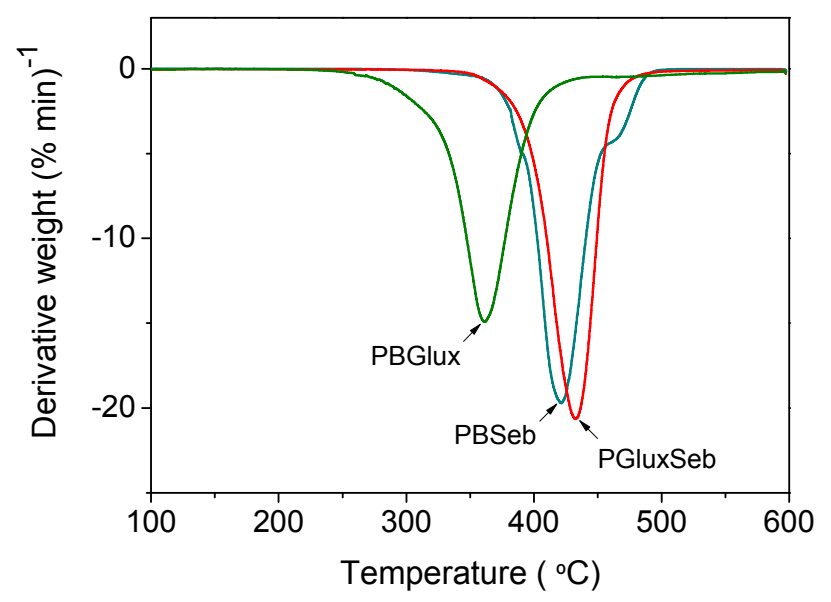

Figure 3. TGA traces of $\mathrm{PB}_{\mathrm{x}} \mathrm{Glux}$ Seb (a) and $\mathrm{PBSeb}_{\mathrm{x}} \mathrm{Glux}_{\mathrm{y}}(\mathrm{b})$, and compared derivative curves for the parent homopolyesters (c). 
temperatures in between those of their two parent homopolyesters and steadily increasing with the amount of butylene units that are replaced by glucitylene ones. These results clearly evidence that the incorporation of Glux-diol units in the PBSeb chain has a significant enhancing effect on its thermal stability.

On the other hand, PBSeb Glux $_{y}$ copolyesters showed an opposite response to heating. In contrast with $\mathrm{PB}_{\mathrm{x}} \mathrm{Glux}$ Seb, the replacement of the sebacate units by the glucarate ones entailed a slight decreasing in both the onset and maximum decomposition rate temperatures, which is fully consistent with the relatively low thermal stability showed by the PBGlux homopolyester. Furthermore, thermal decomposition of these copolyesters was found to proceed along two well differentiated stages with decomposition rates taking place at the proximities of 350 and $410{ }^{\circ} \mathrm{C}$, respectively. The derivative TGA traces revealed that the lower temperature stage evolved as a shoulder with intensity increasing with the glucarate content to become the only peak for the case of PBGlux. It may be reasonably interpreted therefore that the two decomposition steps observed for these copolyesters correspond to the decomposition of glucarate and sebacate sequences, respectively. It is concluded also, that in contrast with the incorporation of Glux-diacid units in the PBSeb chain implies a significant detriment to its thermal stability.

Thermal transitions were characterized by DSC. The heating traces recorded from all the members of the two series of copolyesters are depicted in Figure 4. Endothermal peaks characteristic of melting are observed for the whole $\mathrm{PB}_{\mathrm{x}} \mathrm{Glux} \mathrm{y}$ Seb series with a decreasing trend in both temperature and enthalpy as the content in glucitylene units increases. Particularly worth noting is the case of the homopolyester PGluxSeb which melts at $119^{\circ} \mathrm{C}$, more than $50^{\circ} \mathrm{C}$ above PBSeb. As it will be shown below, the outstanding thermal behavior displayed by this polyester is a consequence of the different structure that it adopts in the crystalline state. The same trend observed for $\mathrm{PB}_{\mathrm{x}} \mathrm{Glux}_{\mathrm{y}} \mathrm{Seb}$ is observed for the PBSeb ${ }_{\mathrm{x}} \mathrm{Glux}_{\mathrm{y}}$ series with the difference that the depressing effect of the incorporation of glucarate units on crystallinity is more 
pronounced than of glucitylene units. In fact, no sign of melting was detected for neither PBGlux nor PBSeb ${ }_{50}$ Glux $_{50}$ and the copolyesters containing $40 \%$ of Glux was unable to crystallize from the melt.

a)

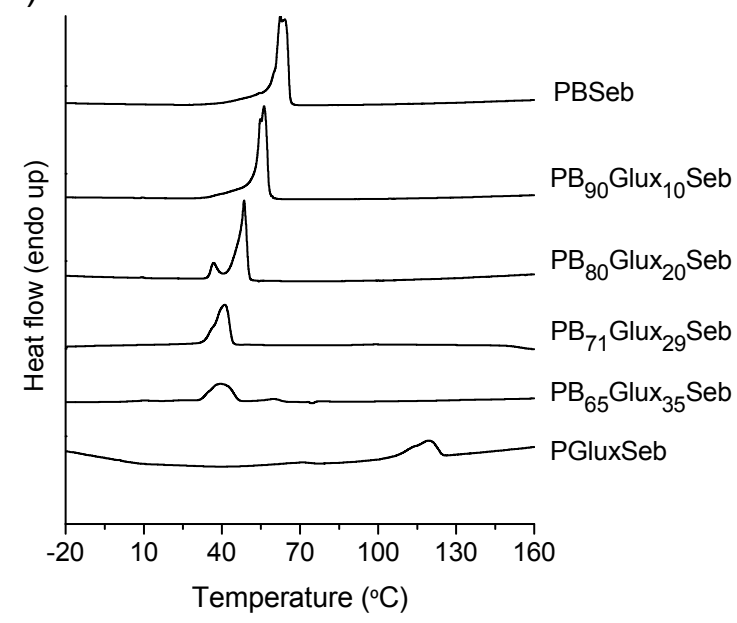

b)

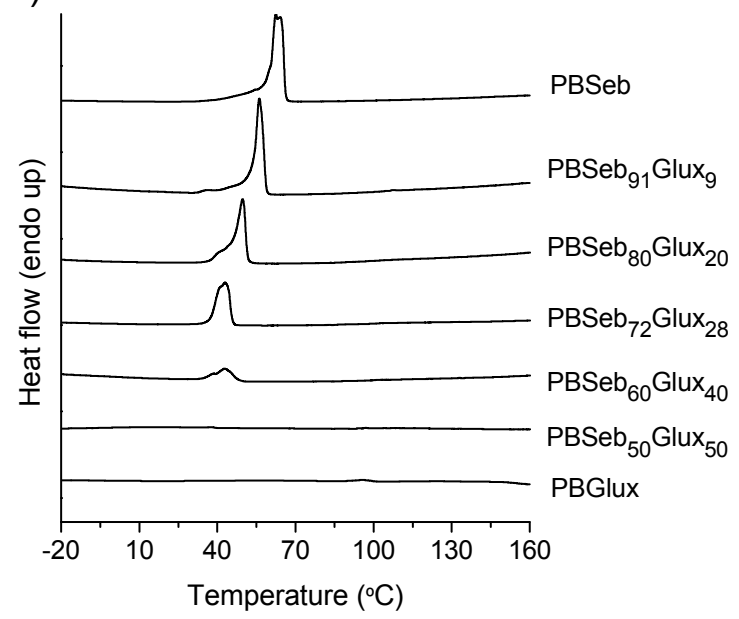

Figure 4. First heating DSC traces of $\mathrm{PB}_{\mathrm{x}} \mathrm{Glux}_{\mathrm{y}} \mathrm{Seb}(\mathrm{a})$ and $\mathrm{PBSeb}_{\mathrm{x}} \mathrm{Glux}_{\mathrm{y}}(\mathrm{b})$ and their parent homopolyesters.

As it is usually done to bring out more conspicuously the DSC trace inflections arising from glass transitions, polyester samples used for $T_{\mathrm{g}}$ measurements were quenched from the melt at $-100{ }^{\circ} \mathrm{C}$ previous to register. The heating thermograms obtained from quenched samples for the two series are depicted in Figure 5 which reveal that polymer crystallization could not be repressed by quenching. In consequence, $T_{\mathrm{g}}$ changes were poorly observed or even unobserved, and data correlation is scarcely reliable given the strong influence of crystallinity on their values. On the other hand, the application of the DDSC method allowed to record $T_{\mathrm{g}}$ values with better definition and for a wider assortment of copolyesters (illustrative DDSC thermograms are provided in the ESI file). The measured $T_{\mathrm{g}}$ values for all the cases in which detection was feasible are given in Table 4. In spite of the difficulty in obtaining the $T_{\mathrm{g}}$ data and the deviations found between the two methods, it can be unquestionably concluded that the changes observed for $T_{\mathrm{g}}$ with composition follow the same upward trend for the two series (Figure 6). Therefore it can be well established 
that a significant increase in $T_{\mathrm{g}}$ of PBSeb is caused by the insertion of the Glux units in the polyester chain whichever is their functionality. The result obtained for the homopolyester PGluxSeb is particularly remarkable. This polyester has a $T_{\mathrm{g}}$ of $28{ }^{\circ} \mathrm{C}$ (almost $90^{\circ} \mathrm{C}$ higher than PBSeb) which combined with its $T_{\mathrm{m}}$ of $119{ }^{\circ} \mathrm{C}$ provides an excellent temperature window for both thermal behavior and processing.

a)

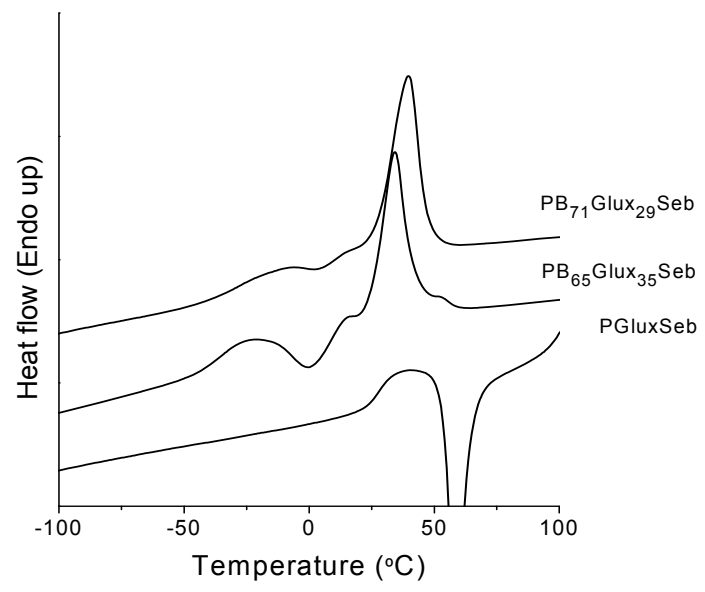

b)

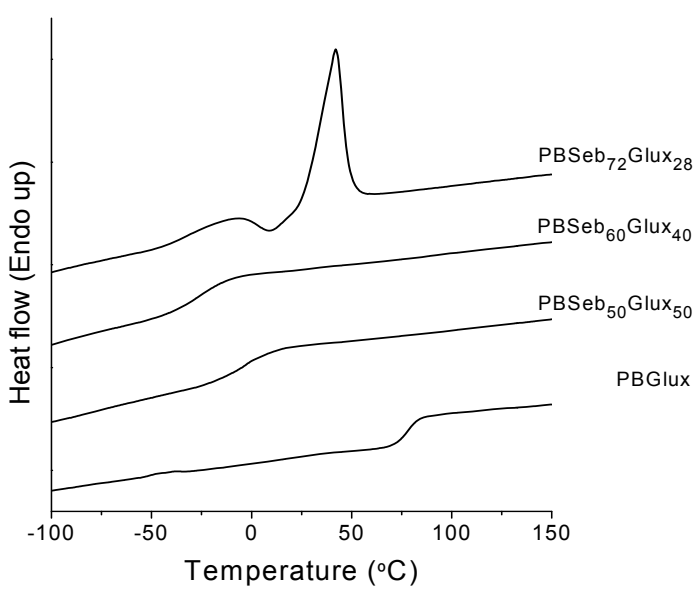

Figure 5. DSC traces of $\mathrm{PB}_{\mathrm{x}} \mathrm{Glux}_{\mathrm{y}} \mathrm{Seb}(\mathrm{a})$ and $\mathrm{PBSeb}_{\mathrm{x}} \mathrm{Glux}_{\mathrm{y}}(\mathrm{b})$ copolyesters quenched from the melt for $T_{\mathrm{g}}$ observation.

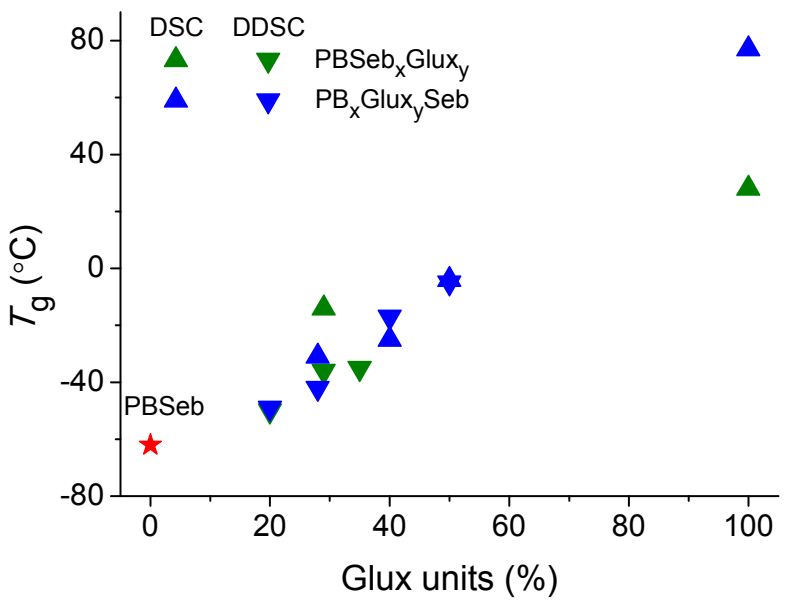

Figure 6. Changes in $T_{g}$ of $\mathrm{PB}_{\mathrm{x}} \mathrm{Glux}_{\mathrm{y}} \mathrm{Seb}$ and PBSeb $\mathrm{Glux}_{\mathrm{y}}$ copolyesters with the content in Glux units. Values for homopolyesters containing $100 \%$ either of Glux-diol or Glux-diacid units are also represented for reference. Note that green and blue colors have been used to differentiate between the series containing Glux-diol and Glux-diacid units, respectively. 
The melting/crystallization and thermal decomposition data for enzymatically and chemically obtained copolyesters for a representative selection of approximately isocompositional pairs from both $\mathrm{PB}_{x} \mathrm{Glux}_{y} \mathrm{Seb}$ and $\mathrm{PBSeb}_{\mathrm{x}} \mathrm{Glux}_{\mathrm{y}}$ series, are given in Table $2 \mathrm{~S}$ in the $\mathrm{SI}$ document. As expected, differences in $T_{\mathrm{m}}$ are small with values differing in less than $3^{\circ} \mathrm{C}$ when copolyester samples crystallized from the melt were compared. Similarly close values were found for decomposition temperatures both onset and maximum rate between the two types of copolyesters. Such small deviations can be readily explained on the basis of differences in either molecular weight or sample history. In fact, the influence of these factors on thermal properties is much more vividly reflected when melting enthalpies are compared. In this case, copolyesters made by enzymes exhibit values that arrive to be up to $100 \%$ greater than those of their corresponding pairs. Such large differences should be interpreted as due mainly to the lower molecular weights that are attained by enzymatic synthesis given the strong influence that chain polymer mobility has on crystallizability.

The crystal structure present in the polyesters containing Glux units was preliminary examined by X-ray diffraction (XRD) and compared with that of PBSeb. The crystal structure adopted by PBSeb has been described in the literature as a rhombic lattice with $a=0.506 \mathrm{~nm}, b=1.456 \mathrm{~nm}$, and $c=2.22 \mathrm{~nm}$ with the polymer chains in alltrans conformation. ${ }^{29}$ The fiber XRD pattern of this structure is distinguished by three very strong reflections, two of them at 4.15 and $3.64 \AA$ arising from the side-by-side packing of the chains, and the third at $22.2 \AA$ corresponding to the axial repeat of the chain in fully extended conformation. The powder XRD patterns recorded from both $\mathrm{PB}_{\mathrm{x}}$ Glux $_{\mathrm{y}}$ Seb and PBSeb $\mathrm{Glux}_{\mathrm{y}}$ copolyesters as well as from PBSeb and PGluxSeb homopolyesters are depicted in Figure 7. All the copolyester profiles display the two sharp reflections at $\sim 3.65$ and $\sim 4.15 \AA$ characteristic of PBSeb which can be taken as a solid proof of that the rhombic structure of the parent homopolyester is retained in the copolyesters regardless their composition. As it has been repeatedly reported for other 
copolyesters containing bicyclic acetalized alditylene or aldarate units, ${ }^{19,21}$ the presence of crystallinity in these systems can be reasonably accounted by assuming that only the homogeneous sequences of PBSeb are integrating the crystal phase whereas the heterogeneous segments containing Glux units are rejected to the amorphous phase. Conversely, the homopolyester PGluxSeb shows a clearly different diffraction profile consisting of broad peaks centered on $15,4.9$ and $4.4 \AA$ indicating that a different crystal structure must be adopted in this case. The larger values observed for the equatorial reflections are consistent with the higher size of the glucitylene unit compared to the butylene one. On the other hand, the spacing observed in the medium angle region at $\sim 15 \AA$ is much shorter than in PBSeb $(\sim 22 \AA)$ which could be interpreted as due to the occurrence of certain shrinkage or even of a helical conformation of the PGluxSeb chain caused by the presence of the skewed cyclic diacetal structure. Nevertheless the poor quality of the PGluxSeb pattern makes this interpretation rather speculative. Obviously further efforts will have to be made in order to clarify this point, which is of doubtless interest regarding the structure-property relationship in Glux containing polyesters. 
a)

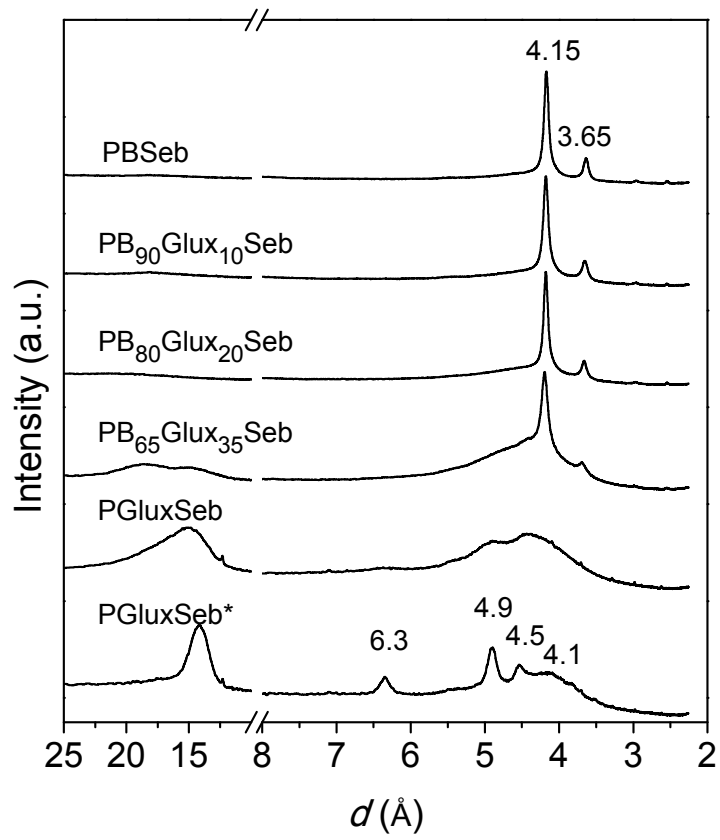

b)

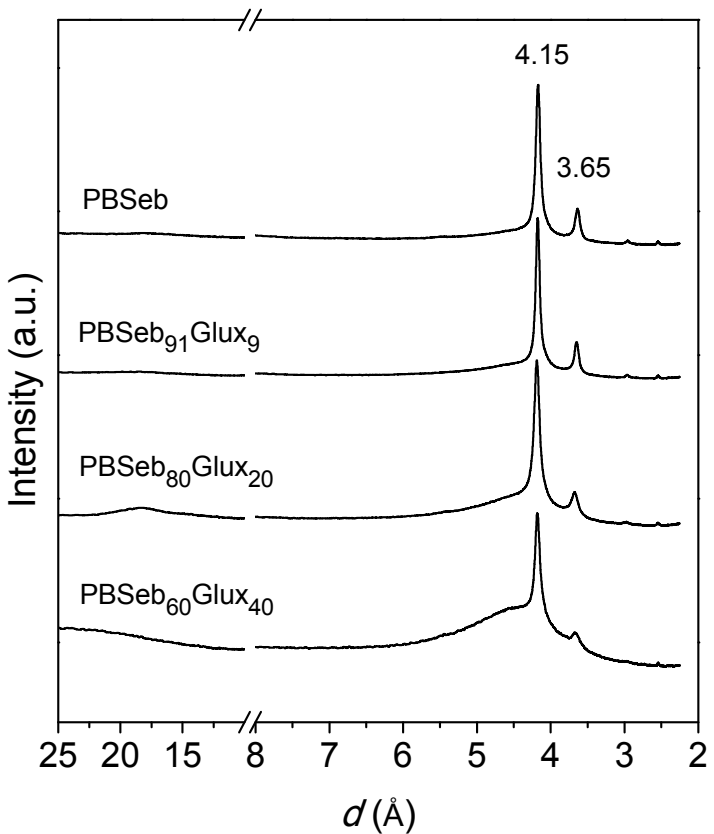

Figure 7. XRD profiles for selected semicrystalline copolyesters of $\mathrm{PB}_{\mathrm{x}} \mathrm{Glux}_{\mathrm{y}} \mathrm{Seb}_{\mathrm{C}}(\mathrm{a})$, $\mathrm{PBSeb}_{\mathrm{x}} \operatorname{Glux}_{\mathrm{y}}$ (b) and their parent homopolyesters. Diffractograms were recorded from powder samples without any previous thermal treatment. *Profile obtained from PGluxSeb annealed at $110^{\circ} \mathrm{C}$ for 2 hours.

\subsection{Biodegradation and hydrolytic degradability}

To evaluate the effects that the incorporation of Glux (glucitylene or glucarate) units exert on the biodegradability and hydrolytic degradability of PBSeb, the PB ${ }_{90}$ Glux $_{10}$ Seb, PB $_{71}$ Glux $_{29}$ Seb, PBSeb ${ }_{91}$ Glux $_{9}$ copolyesters obtained by melt polycondensation were selected in addition to PBSeb itself for a comparative degradation study. $\mathrm{PB}_{90} \mathrm{Glux}_{10} \mathrm{Seb}$ and $\mathrm{PB}_{71} \mathrm{Glux}_{29} \mathrm{Seb}$ belong to the series in which the diol unit is replaced but they largely differ in composition. PBSeb $_{91}$ Glux $_{9}$ is a poly(sebacate-co-glucarate) copolyester that has approximately the same content in Glux units as $\mathrm{PB}_{90} \mathrm{Glux}_{10} \mathrm{Seb}$. 
The study was first addressed to evaluate the biodegradability of the Gluxcontaining copolyesters. The degradation experiments were carried out by incubating the copolyesters in aqueous $\mathrm{pH} 7.4$ buffer at $37^{\circ} \mathrm{C}$ with and without porcine pancreas lipase for four weeks. The changes taking place in sample weights and molecular weights of the samples along the period of incubation are shown in the two plots depicted in Figure 8.

a)

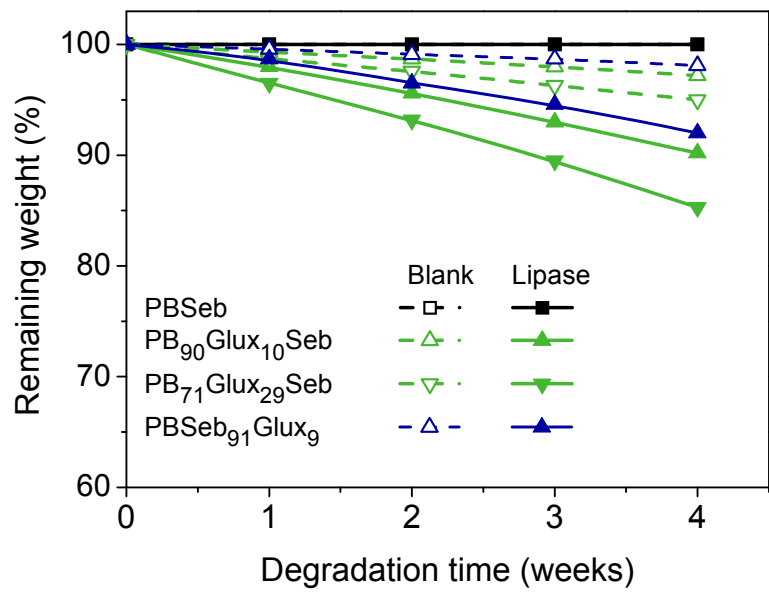

b)

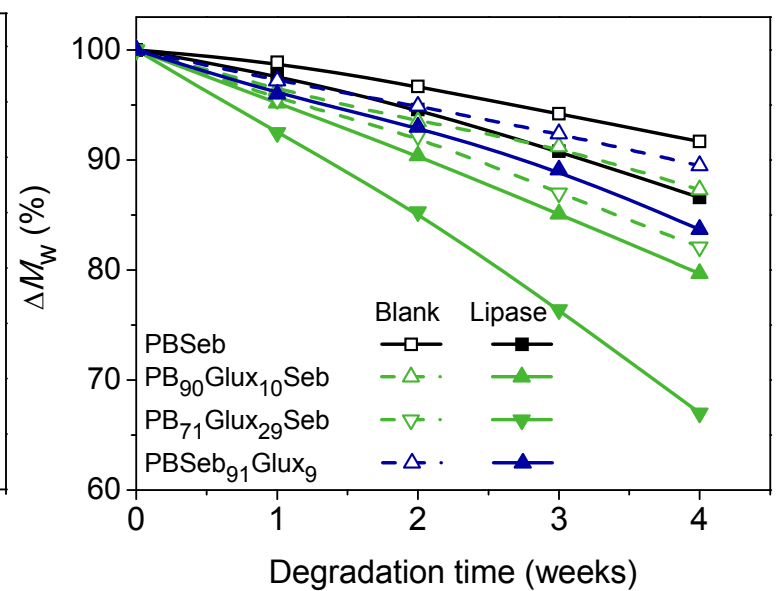

Figure 8. Enzymatic degradation of selected polyesters upon incubation at $\mathrm{pH} 7.4$ at $37^{\circ} \mathrm{C}$ for four weeks. Remaining weight (a) and molecular weight (b) vs. degradation time.

After 4 weeks of incubation in the presence of porcine pancreas enzyme PB $_{90}$ Glux $_{10}$ Seb, PB $_{71}$ Glux $_{29}$ Seb, PBSeb ${ }_{91}$ Glux $_{9}$ copolyesters lost around 10, 15 and $8 \%$ of their initial weight, respectively, whereas no changes were perceivable for PBSeb. Conversely, the weight losses observed for all copolyesters when incubated in the absence of lipase was between 3 and $5 \%$. The evolution of molecular weight along incubation time follows a similar trend but changes were now more noticeable. Although no mass loss was perceived for PBSeb, the GPC analysis revealed that this polyester was partially degraded by the enzyme. Such differences between the two plots are likely due to the limited solubility of the degradation products in the incubation medium that makes them to remain attached to the sample. 
A simple comparison of the degradation curves leads to conclude that the biodegradabilty of PBSeb is increased by the insertion of Glux units whatever their nature and that such effect increases with the content in such units. It is worth of mention the similar behavior displayed by the two approximately isocompositional copolyesters $\mathrm{PB}_{90} \mathrm{Glux}_{10} \mathrm{Seb}$ and $\mathrm{PBSeb}_{91} \mathrm{Glux}_{9}$ that evidences the irrelevance of the type of Glux unit as far as biodegradability is concerned. This result is contrary to previous observations carried out on other copolyesters containing cyclic sugar-based units which displayed a degradation behavior strongly dependent on the function (diol or diacid) of the replaced unit. Thus poly(butylene terephthalate) copolyesters containing Glux units were reported to show a largely enhanced degradability only in the case that the sugar-based units were replacing the terephthalate units. ${ }^{22 b}$ The same was reported for PBT containing Galx units (bicyclic diacetalized units derived from Dgalactose) ${ }^{30}$ It seems therefore that it is not only the function but also the chemical structure of the replaced unit that plays a determinant role on the influence of composition on the biodegradability of the copolyester.

As a second part of the study on the degradability of Glux containing PBSeb copolyesters, the same set selected for biodegradability was then incubated under rather aggressive conditions, i.e. aqueous buffer $\mathrm{pH} 2$ at $37{ }^{\circ} \mathrm{C}$ to evaluate their resistance to hydrolysis. The changes in sample weight and molecular weight undergone by the polyesters along incubation are shown in the plots depicted in Figure 9. After four weeks of treatment the weight losses observed were 7, 10 and $6.6 \%$ of their initial mass for $\mathrm{PB}_{90} \mathrm{Glux}_{10} \mathrm{Seb}, \mathrm{PB}_{71} \mathrm{Glux}_{29} \mathrm{Seb}$ and $\mathrm{PBSeb}_{91} \mathrm{Glux}_{9}$ respectively, whereas no change at all was noticed for PBSeb. As before, changes in molecular weight were more noticeable than mass loses and a decrease of about $15 \%$ of the original value was observed for PBSeb. The conclusions that can be drawn from these essays regarding hydrolysis-composition dependence of the copolyesters are in line with those drawn from the biodegradation study although differences among them are now more attenuated. What is really striking is the fact that these copolyesters 
degraded faster at $\mathrm{pH} 7$ in the presence of lipases than at $\mathrm{pH} 2$ in absence of enzyme. This is contrary not only to what happens in PBSeb but also to all observations previously reported on sugar containing copolyesters. ${ }^{22,30}$

a)

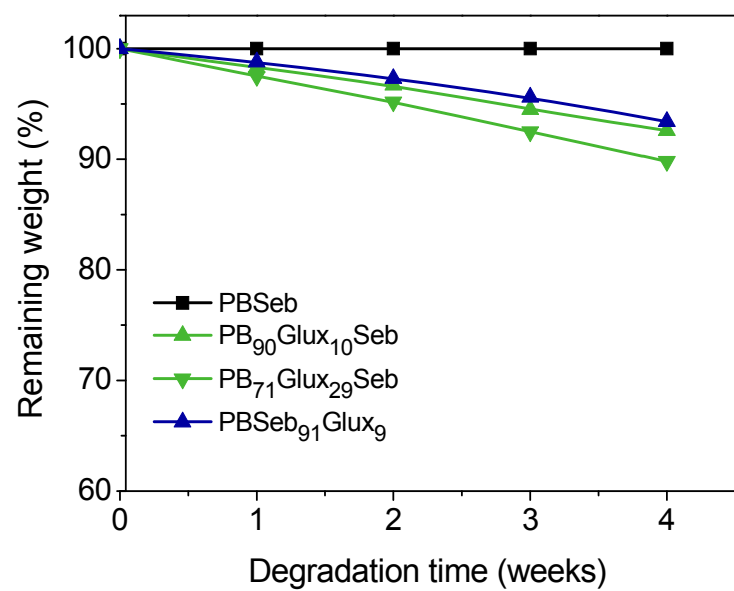

b)

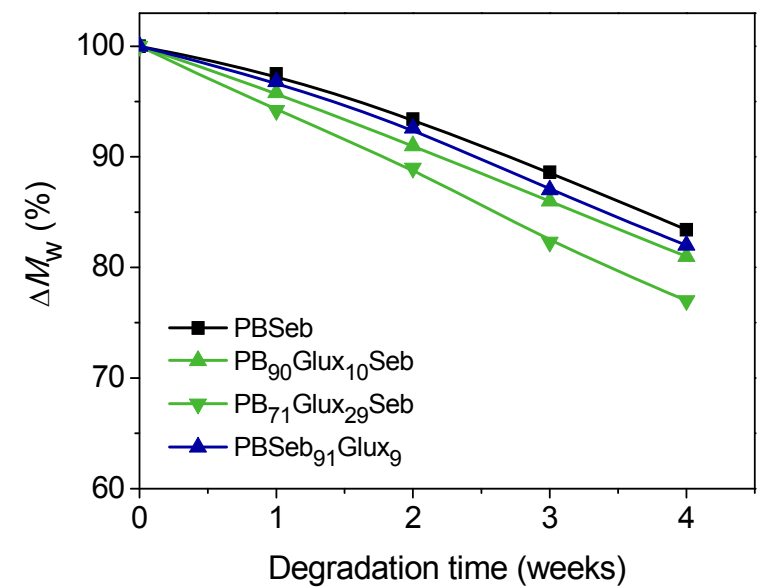

Figure 9. Hydrolytic degradation of selected polyesters at $37^{\circ} \mathrm{C}, \mathrm{pH}$ 2. Remaining weight (a) and molecular weight (b) vs. degradation time.

Finally, the morphological changes taking place on the samples due to degradation were brought into evidence by SEM. Illustrative pictures for $\mathrm{PB}_{90} \mathrm{Glux}_{10} \mathrm{Seb}$ are shown in Figure 10, and those for $\mathrm{PB}_{71} \mathrm{Glux}_{29} \mathrm{Seb}$ and $\mathrm{PBSeb}_{91} \mathrm{Glux}_{9}$ copolyesters and PBSeb are provided in the ESI file. These pictures confirm the results obtained by weighing and GPC of the degraded samples, and illustrate that a different degradation mechanism seems to be followed depending on incubation conditions. As it is seen in Figure 10c, almost no changes are observed in the sample incubated at $\mathrm{pH} 7.4$ in the absence of enzymes, which is in agreement with the small changes found in the analysis (Figure 8). On the contrary, plenty of small holes are seen on the sample subjected to the action of lipases (Figure 10e), which evidences the susceptibility of $\mathrm{PB}_{90} \mathrm{Glux}_{10}$ Seb to a bulk enzymatic degradation. Figure $10 \mathrm{~b}$ shows the copolyester sample after incubation at $\mathrm{pH} 2$. In this case the surface of the incubated sample appears to be much coarser than original reflecting the attack of the acidic medium but 
holes are barely perceivable; this morphology indicates that an erosion mechanism is probably operating in the degradation of the copolyester under these conditions.

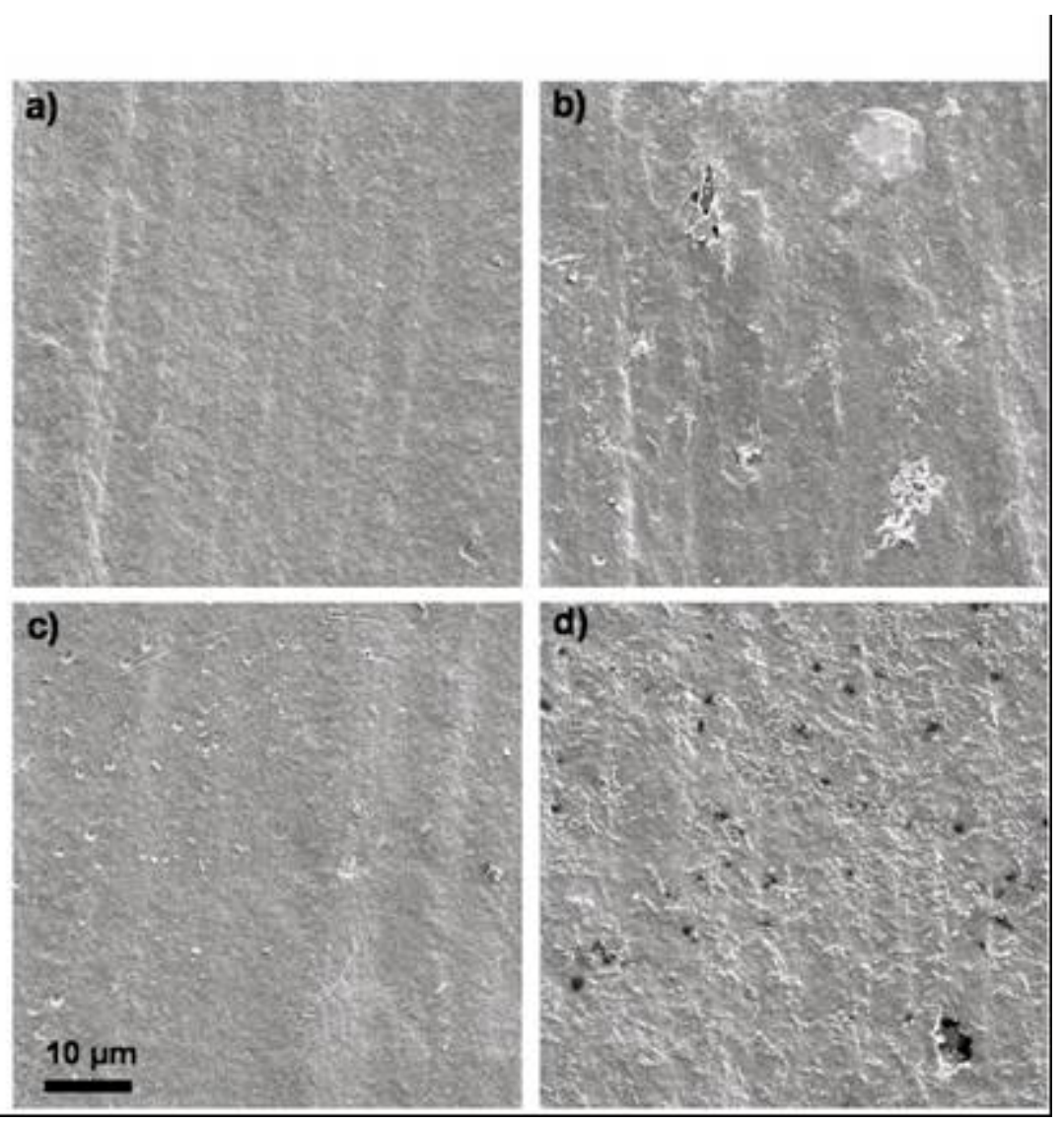

Figure 10. SEM micrographs of $\mathrm{PB}_{90} \mathrm{Glux}_{10} \mathrm{Seb}$. Initial sample (a) and after incubation for four weeks at pH 2 (b), pH 7.4 without lipases (c), and pH 7.4 in the presence of lipases (d).

\section{Conclusions}

A set of bio-based compounds has been successfully used as platform for the synthesis of lineal aliphatic copolyesters by polycondensation both in the melt and in solution assisted by CALB. Mixtures of 1,4-butanediol, diethyl sebacate and the bicyclic methylene acetal of either D-glucitol (Glux-diol) or dimethyl D-glucarate (Glux-diester) were combined to produce two series of copolyesters (PB $\mathrm{PBlux}_{\mathrm{y}} \mathrm{Seb}$ and PBSeb $\mathrm{Glux}_{\mathrm{y}}$ ) containing up to $50 \%$ of sugar-based units and differing to each other in which form (diol or diacid) are the Glux units incorporated in the copolymer. Also the three parent homopolyesters (PBSeb, PBGlux and PGluxSeb) were prepared by both procedures. 
The enzymatic synthesis has proven to be effective to afford polyesters with $M_{\mathrm{w}} \sim 10,000$ whereas $M_{\mathrm{w}}$ up to near 40,000 could be attained by melt polycondensation. In both cases the molecular weight of the copolyesters decreased with the content in Glux units and their microstructure was almost random for whichever composition.

The insertion of Glux units in PBSeb had noticeable effects on the properties of the polyester depending not only on their content but also on their functionality (diol or diacid). $\mathrm{PB}_{\mathrm{x}}$ Glux $\mathrm{y}$ Seb copolyesters are more resistant to heat than PBSeb but on the contrary, the thermal stability of the PBSeb $\mathrm{Glux}_{\mathrm{y}}$ series is slightly lower. Both crystallinity and melting temperature of PBSeb were depressed upon insertion of Glux units whether they are diol or diacid but the effect was more pronounced in the PBSeb $_{x}$ Glux $_{y}$ series. Furthermore, the crystal structure of PBSeb was preserved in the two copolyester series. Although glass transitions were difficult to detect in these copolyesters, accessible data were able to reveal a notable increase in $T_{\mathrm{g}}$ with the content in Glux units. This is an outstanding result since a too low $T_{\mathrm{g}}$ value is one of the main drawbacks of aliphatic polyesters. Lastly, the degradability of PBSeb was enhanced by the presence of Glux units; not only the chemical hydrolysis was hastened but also the sensitivity to enzymatic degradation was significantly increased.

PGluxSeb made exclusively from Glux-diol and diethyl sebacate is a new homopolyester that deserves particular attention. Sebacic acid and D-glucose are the raw materials needed for producing this polymer. In spite of being obtained with a rather low molecular weight $\left(M_{\mathrm{w}} / M_{\mathrm{n}}=11,200 / 5,600\right)$, it appears to be more thermally stable than PBSeb, has melting and glass-transition temperatures of 119 and $28{ }^{\circ} \mathrm{C}$, respectively, and it is able to crystallize from the melt with almost full reproducibility of its initial crystallinity. Further efforts addressed to attain this polyester with higher molecular weight should be made in order to estimate their optimal properties and to evaluate properly its potential as a partially biodegradable bio-based thermoplastic with interest as biomaterial. 


\section{Supporting Information Available}

Figures of 1D and 2D NMR spectra, derivative TGA traces, DDSC traces and SEM pictures of some copolyesters are supplied. Tables containing additional enzymatic polymerization data and thermal properties of some enzymatically synthesized copolyesters are also provided. This information is available free of charge via the Internet at http://pubs.acs.org/.

\section{Acknowledgements}

Financial support for this work was provided by MINECO (Spain) with Grant MAT-2012-38044-CO3-03, and by AGAUR (Catalonia) with grant 2009SGR1469. The help of Prof. X. Ramis with $T_{\mathrm{g}}$ determination is greatly acknowledged. Authors are also indebted to MINECO (Spain) for the FPI grant awarded to Cristina Japu.

\section{References}

1. a) Belgacem, M.N.; Gandini, A. Monomers, Polymers and Composites from Renewable Resources, Elsevier Science, 2011.; b) Gandini, A. Green Chem. 2011, 13, 1061-1083.

2. Albertsson, A.C.; Varma, I. Adv. Polym. Sci. 2002, 157, 1-40.

3. Chen, G.Q.; Patel, M.K. Chem. Rev. 2012, 112, 2082-2099.

4. Miller, S.A. ACS Macro Lett. 2013, 2, 550-554.

5. Wang, H.; Xu, M.; Wu, Z.; Zhang, W.; Ji, J.; Chu, P.K. ACS Appl. Mater. \& Interfaces 2012, 4, 4380-4386.

6. Coutinho, D.F.; Gomes, M.E.; Neves, N.M.; Reis, R.L. Acta Biomater. 2012, 8, 1490-1497.

7. Kim, J.; Lee, K.W.; Hefferan, T.E.; Currier, B.L.; Yaszemski, M.J.; Lu, L. Biomacromolecules 2007, 9, 149-157.

8. Minh, D.; Besson, M.; Pinel, C.; Fuertes, P.; Petitjean, C. Topics in Catalysis 2010, 53, 1270-1273.

9. Mutlu, H.; Meier, M.A.R. Eur. J. Lipid Sci. Tech. 2010, 112, 10-30. 
10. a) Shikanov A.; Domb, A.J. Biomacromolecules, 2006, 7, 288-296; b) Shikanov, A.; Vaisman, B.; Shikanov, S.; Domb, A.J. J. Biomed. Mater. Res., Part A 2010, 92A, 1283-1291.

11. a) Dang, W.; Daviau, T.; Ying, P.; Zhao, Y.; Nowotnik, D.; Clow, C.S.; Tyler, B.; Brem, H.; J. Control. Release 1996, 42, 83-92; b) Domb, A.J.; Rock, M.; Perkin, C.; Yipchuck, G.; Broxup, B.; Villemure, J.G. Biomaterials 1995, 16, 1069-1072.

12. Motlagh, D.; Yang, J.; Lui, K.Y.; Webb, A.R.; Ameer, G.A. Biomaterials 2006, 27, 4315-4324.

13. a) Bastioli, C.; Borsotti, G.; Capuzzi, L.; Vallero, R. US 2011/0071238; b) Jacquel, N.; Saint-Loup, R.; Fenouillot-Rimlinger, F.; Pascault, J.-P.; Rousseau, A. WO2013/144525.

14. Mortensen, P.B.; Gregersen, N. Biochim. Biophys. Acta (BBA), Lipids and Lipid Metabolism 1982, 710, 477-484.

15. Liang, Y.; Xiao, L., Zhai, Y.; Xie, C.; Deng, L.; Dong, A. J. Appl. Polym. Sci. 2013, 127, 3948-3953.

16. Burdick, J.A.; Mauck, R.L. Biomaterials for Tissue Engineering Applications: $A$ Review of the Past and Future Trends, Springer, 2010, p. 98.

17. Terada, S.; Takagi J. (Mitsubishi Plastics, Inc.), US6960374 B1, 2005.

18. Gohil, R.M.; Tao, Y.; Teasley, M.F.; Wilczek, L. (E. I. Du Pont De Nemours And Company), WO2013101992 A1, 2013

19. Bland, R.; Bradley, J.; Jonza, J.; Smith, J.; Smith, K. (Minnesota Mining \& MFG), EP0591055, 1998.

20 Harmon, K.K. (Minnesota Mining and Manufacturing Company), US5304224 A, 1994.

21. Freese, F.; Loos, R.; Keck, J.; Auffermann, J.; Yang, X. (Basf Se), US20120288650 A1, 2012.

22. a) Japu, C.; Martínez de llarduya, A.; Alla, A.; García-Martín, M.G.; Galbis, J.A.; Muñoz-Guerra, S. Polym. Chem. 2013, 4, 3524-3536; b) Japu, C.; Martínez de Ilarduya, A.; Alla, A.; García-Martín, M.G.; Galbis, J.A.; Muñoz-Guerra, S. Polym. Chem. 2014, 5, 3190-3202; c) Japu, C.; Alla, A.; Martínez de llarduya, A.; García-Martín, M.G.; Benito, E.; Galbis, J.A.; Muñoz-Guerra, S. Polym. Chem. 2012, 3, 2092-2101.

23. a) Dhamaniya, S.; Jacob, J. Polymer 2010, 51, 5392-5399: b) Dhamaniya, S.; Jacob, J. Polym. Bull. 2012, 68, 1287-1304. 
24. a) Lavilla, C.; Alla, A.; Martínez de Ilarduya, A.; Benito, E.; García-Martín, M.G.; Galbis, J.A.; Muñoz-Guerra, S. Biomacromolecules 2011, 12, 2642-2652; b) Lavilla, C.; Alla, A.; Martínez de llarduya, A.; Muñoz-Guerra, S. Biomacromolecules 2013, 14, 781-793.

25. Azim, H.; Dekhterman, A.; Jiang, Z.Z.; Gross, R.A. Biomacromolecules 2006, 7, 3093-3097.

26. Jiang, Y.; Woortman, A.J.J.; van Ekenstein, G.O.R.A.; Loos, K. Biomolecules 2013, 3, 461-480.

27. Kong, X. Qi, H.; Curtis, J.M., J. Appl. Polym. Sci. 2014, DOI:10.1002/APP.40579.

28. Ahn, T.O.; Lee, M.; Jeong H. M.; Cho, K. J. Polym. Sci. Part B: Polym. Phys. 1995, 33, 327-329.

29. Almontassir, A.; Gestí, S.; Franco, L.; Puiggalí, J. Macromolecules 2004, 37, 5300-5309.

30. Lavilla, C.; Muñoz-Guerra, S. Polym. Degrad. Stab. 2012, 97, 1762-1771.

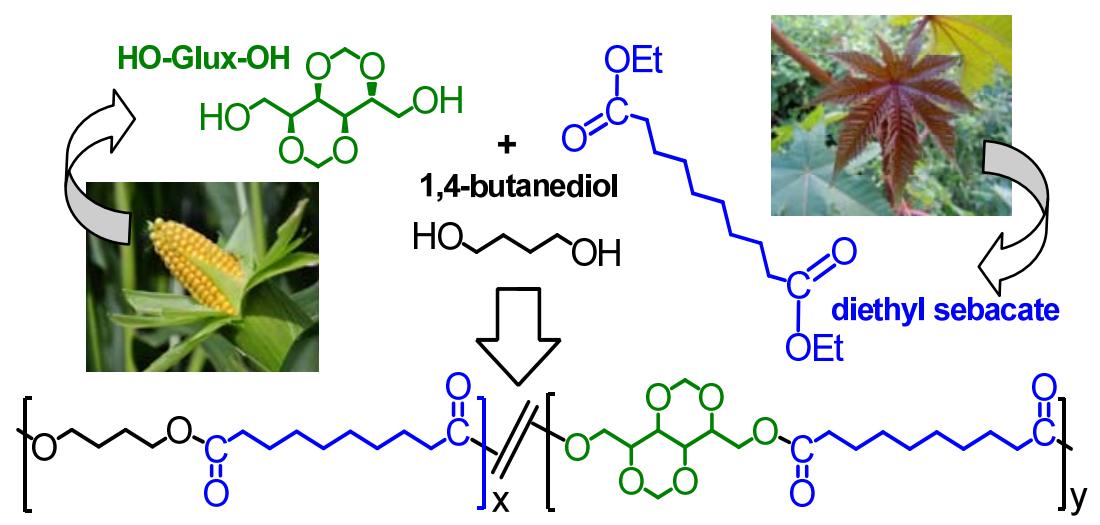

PBS copolyesters containing Glux units

\section{Table of contents}

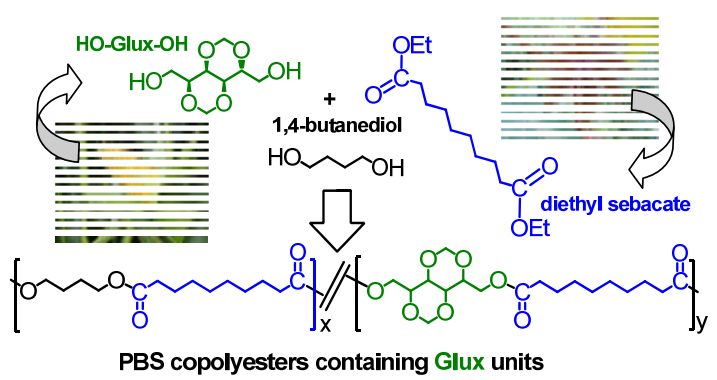

Sustainable copolyesters made from 1,4-butanediol, sebacic acid and D-glucose by melt and enzymatic polycondensation

C. Japu, A. Martínez de llarduya, A. Alla, Y. Jiang,

K. Loos and S. Muñoz-Guerra 
Supporting information

\title{
Copolyesters made from 1,4-butanediol, sebacic acid and D-glucose by melt and enzymatic polycondensation
}

\author{
Cristina Japu, ${ }^{a}$ Antxon Martínez de llarduya, ${ }^{a}$ Abdelilah Alla ${ }^{a}$ \\ Yi Jiang, ${ }^{b}$ Katja Loos $^{b}$ and Sebastián Muñoz-Guerra ${ }^{a^{*}}$ \\ aUniversitat Politècnica de Catalunya, ETSEIB, Diagonal 647, 08028 Barcelona, Spain \\ bernike Institute for Advanced Materials, University of Groningen, Nijenborgh 4, 9747 AG \\ Groningen, The Netherlands
}




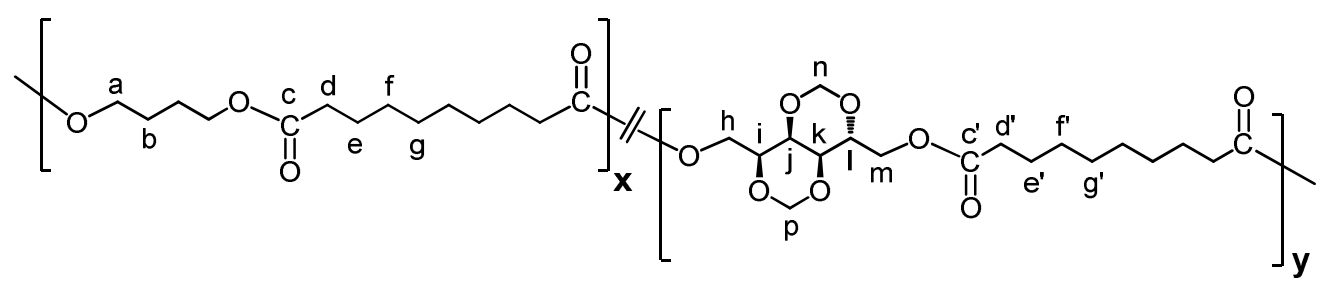
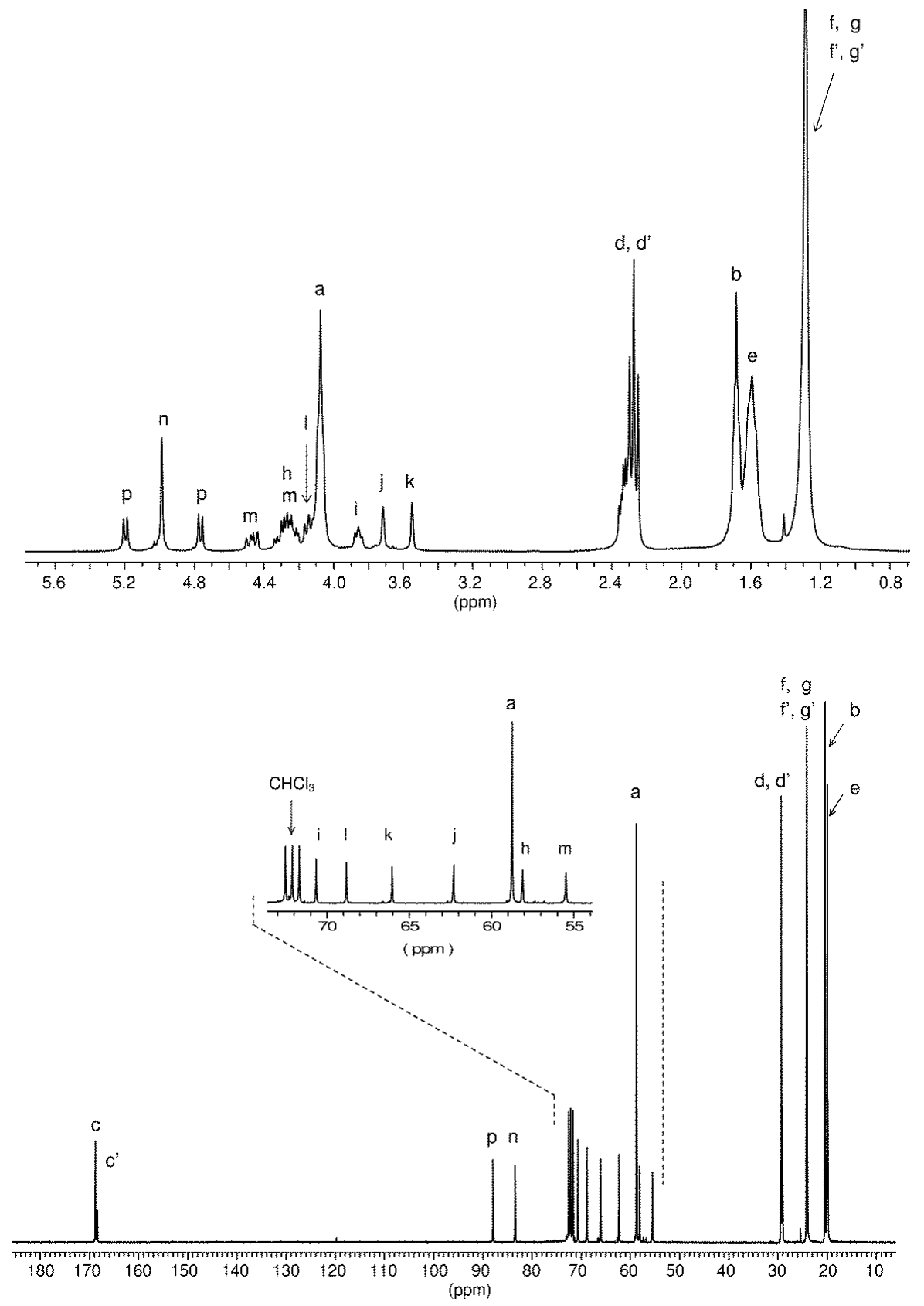

Figure S1. ${ }^{1} \mathrm{H}$ (top) and ${ }^{13} \mathrm{C}$ (bottom) NMR spectra of $\mathrm{PB}_{71} \mathrm{Glux}_{29}$ Seb copolyester. 

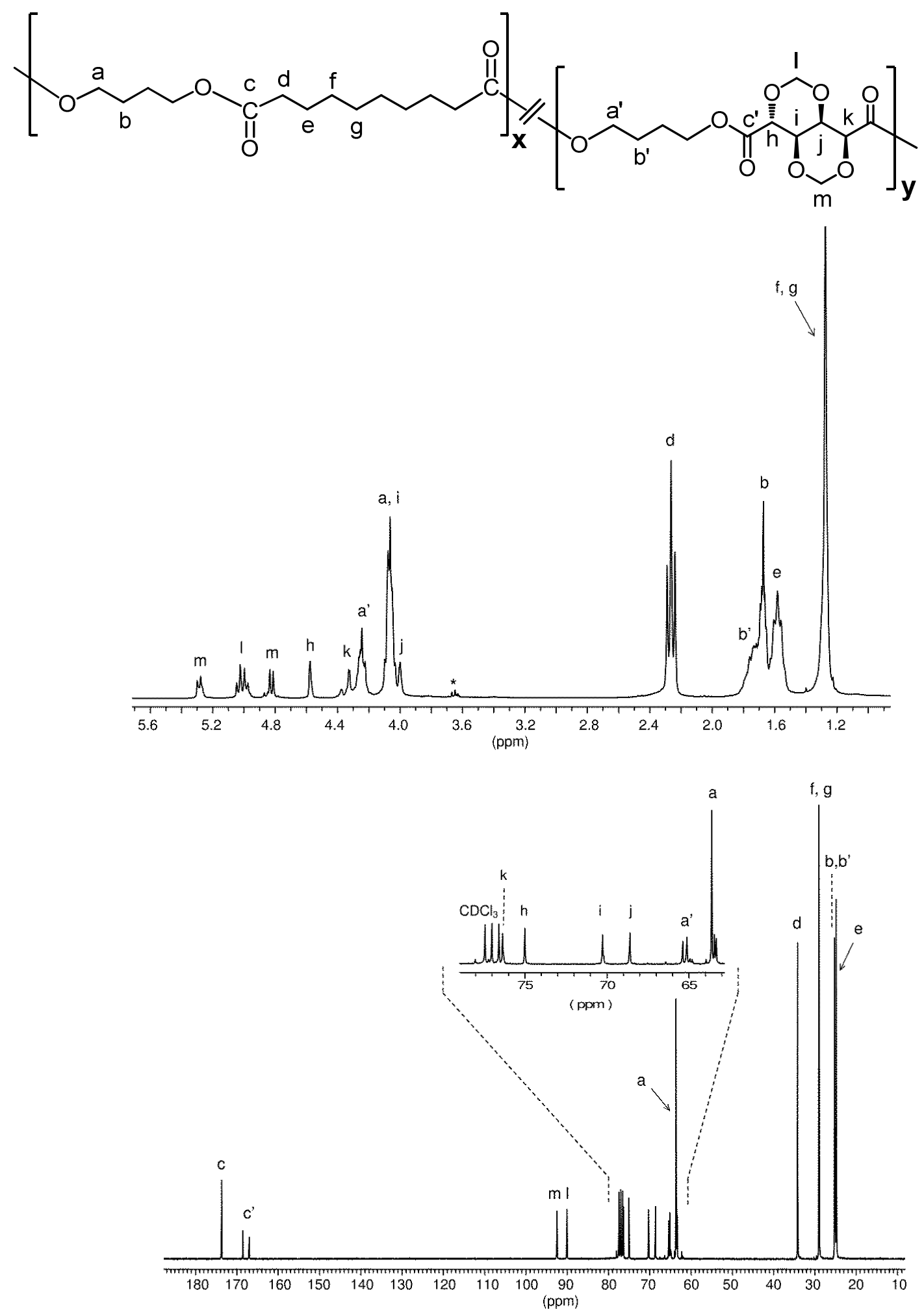

Figure S2. ${ }^{1} \mathrm{H}$ (top) and ${ }^{13} \mathrm{C}$ (bottom) NMR spectra of $\mathrm{PBSeb}_{72} \mathrm{Glux}_{28}$ copolyester. 

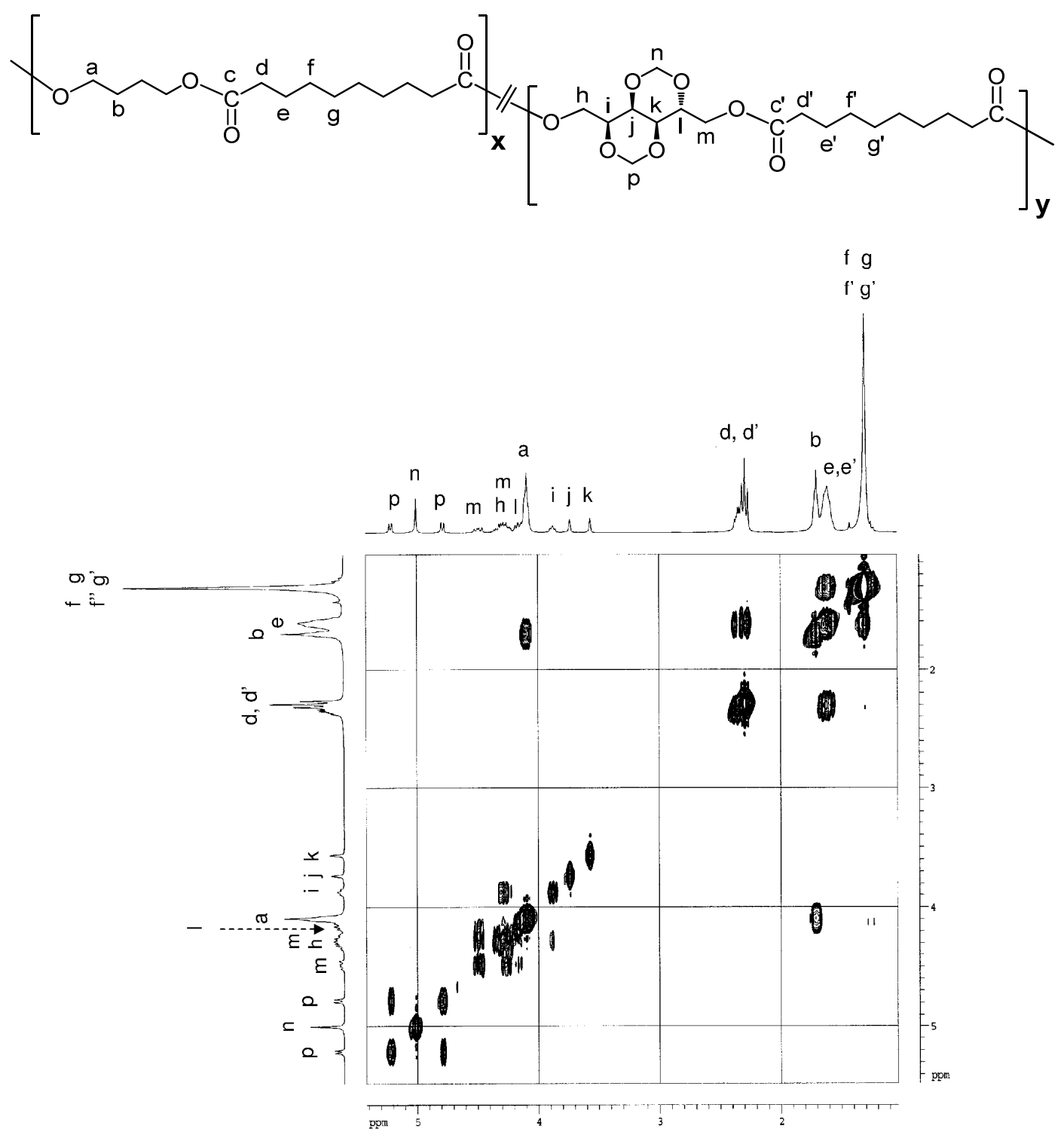

Figure S3. COSY ${ }^{1} \mathrm{H}-{ }^{1} \mathrm{H}$ NMR spectrum of $\mathrm{PB}_{71} \mathrm{Glux}_{29}$ Seb copolyester. 

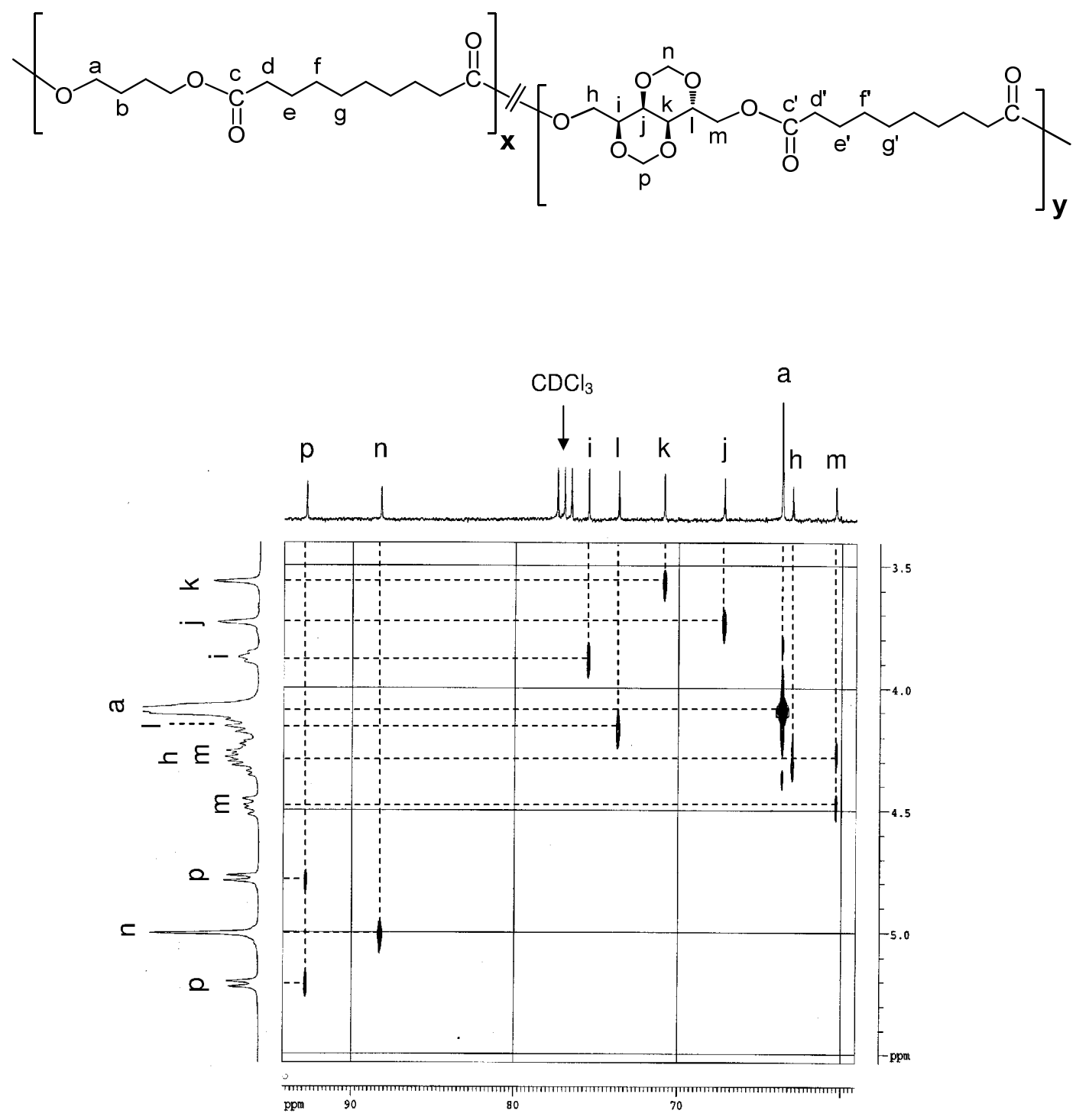

Figure S4. ${ }^{1} \mathrm{H}-{ }^{13} \mathrm{C}$ HETCOR NMR spectrum of $\mathrm{PB}_{71} \mathrm{Glux}_{29} \mathrm{Seb}$ copolyester (enlarged region). 


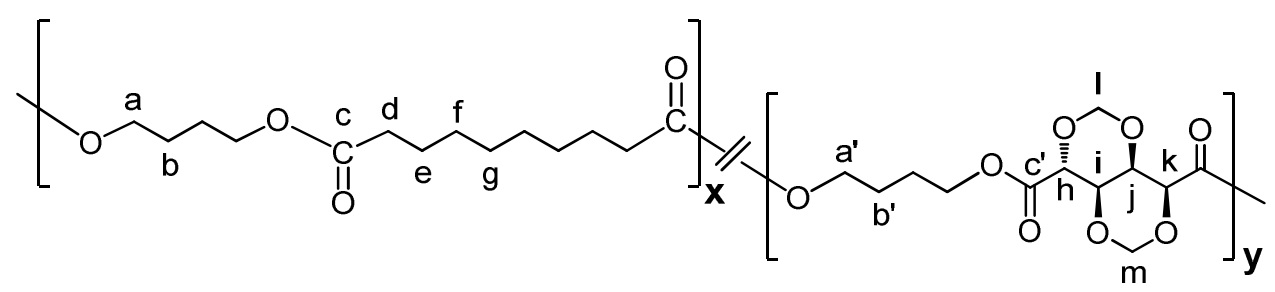

f, g

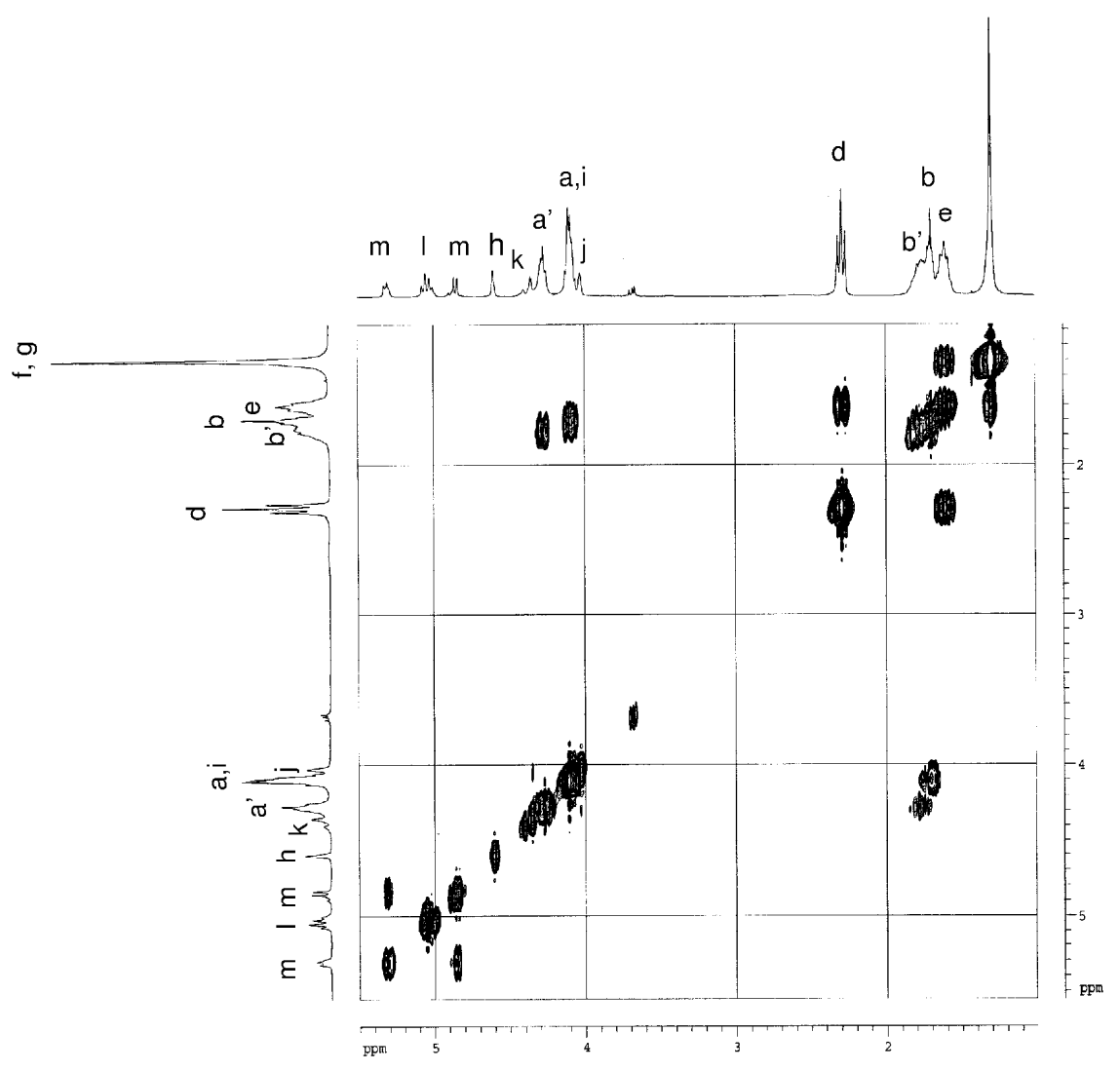

Figure S5. COSY ${ }^{1} \mathrm{H}-{ }^{1} \mathrm{H}$ NMR spectrum of $\mathrm{PBSeb}_{72} \mathrm{Glux}_{28}$ copolyester. 

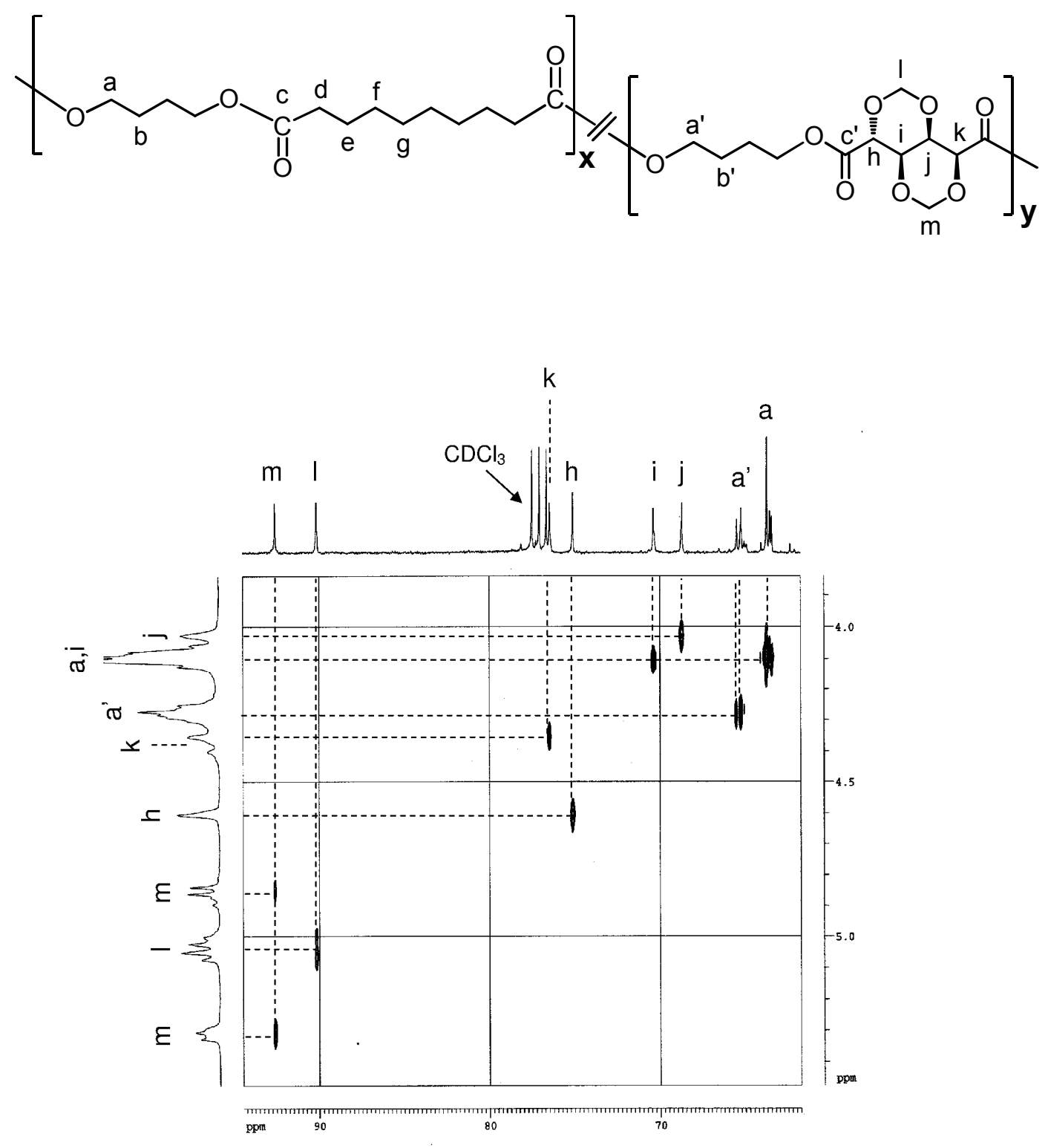

Figure S6. ${ }^{1} \mathrm{H}-{ }^{13} \mathrm{C}$ HETCOR NMR spectrum of $\mathrm{PBSeb}_{72} \mathrm{Glux}_{28}$ copolyester (enlarged). 


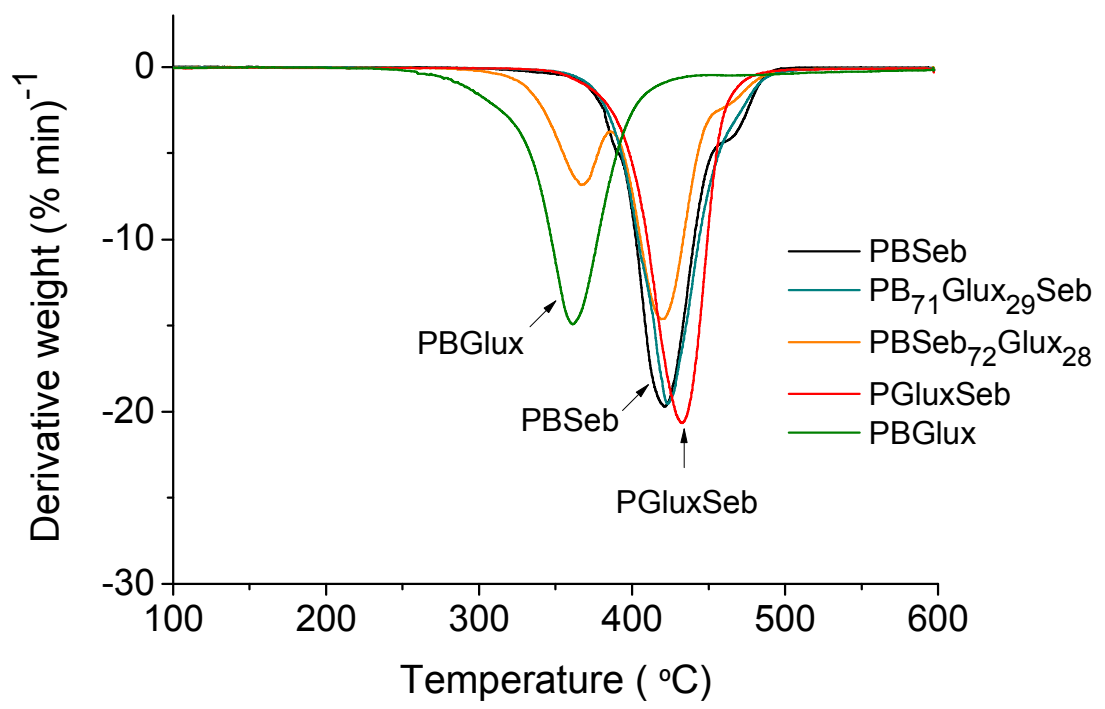

Figure S7. TGA compared derivative curves for a selection of cases.

a)

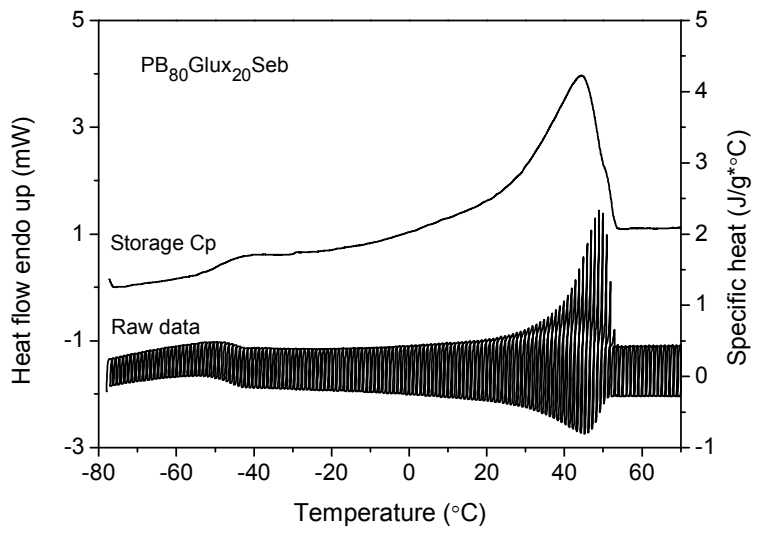

b)

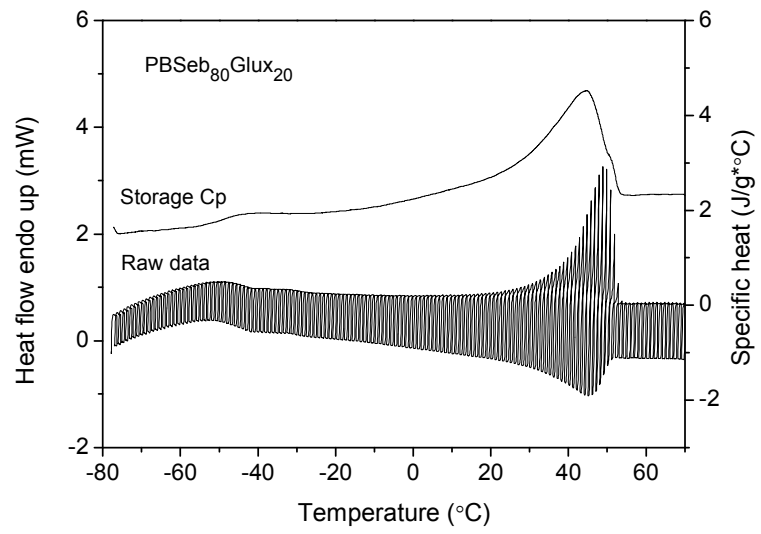

Figure S8. DDSC traces of the indicated copolyesters. 

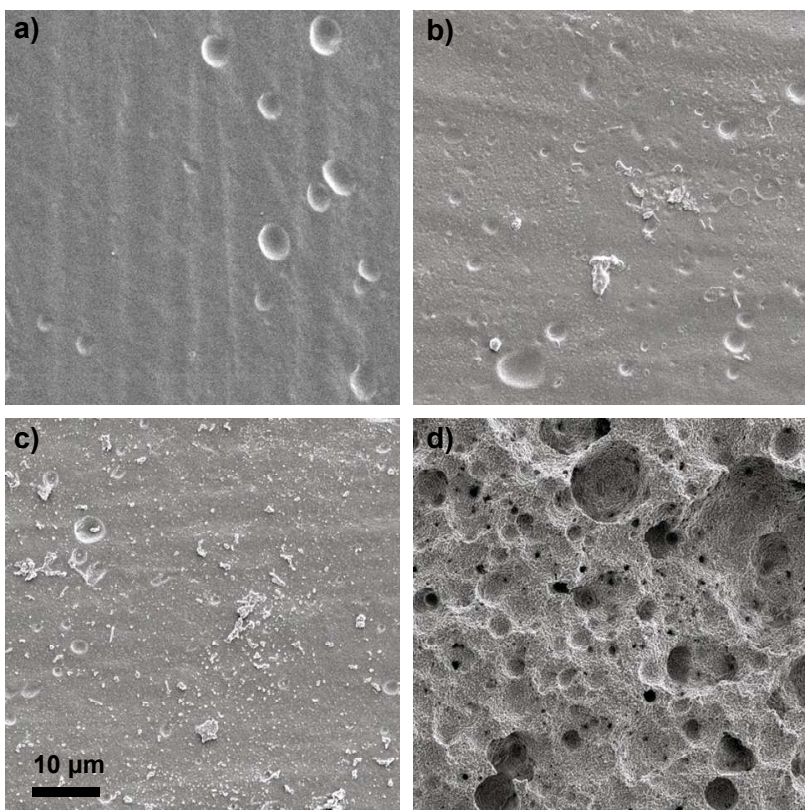

Figure S9. SEM micrographs of $\mathrm{PB}_{71} \mathrm{Glux}_{29}$ Seb: initial sample (a); after incubation at $\mathrm{pH} 2$ for four weeks (b); after incubation at $\mathrm{pH} 7.4$ for four weeks without (c) and with (d) porcine pancreas lipase.
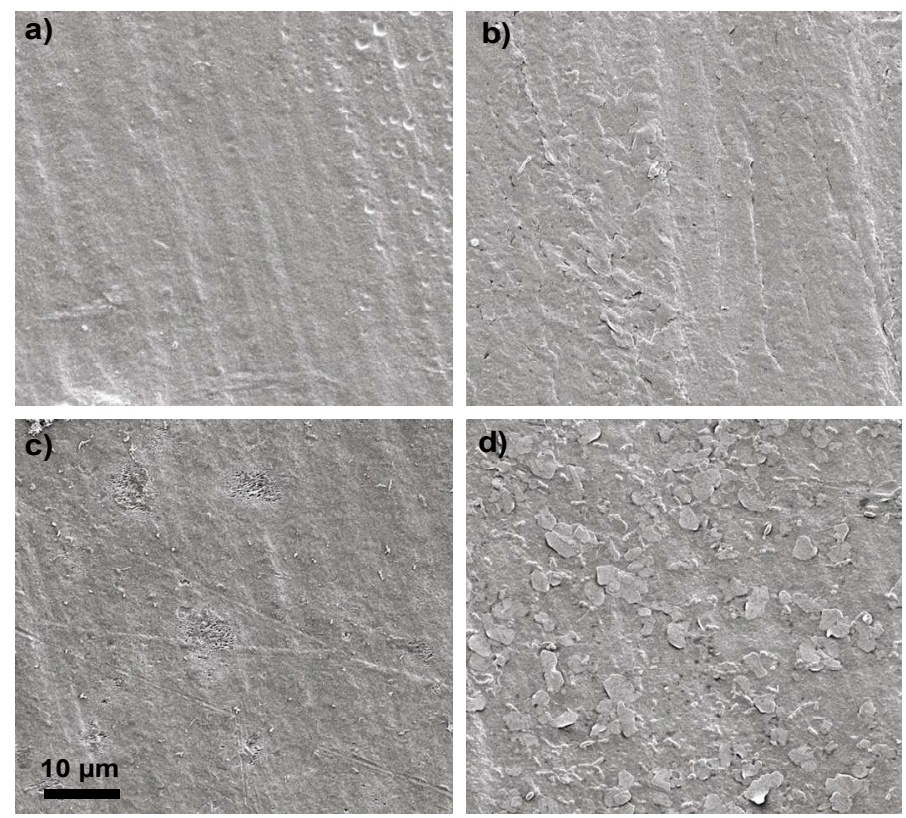

Figure S10. SEM micrographs of PBSeb: initial sample (a); after incubation at $\mathrm{pH} 2$ for four weeks (b); after incubation at $\mathrm{pH} 7.4$ for four weeks without (c) and with (d) porcine pancreas lipase. 

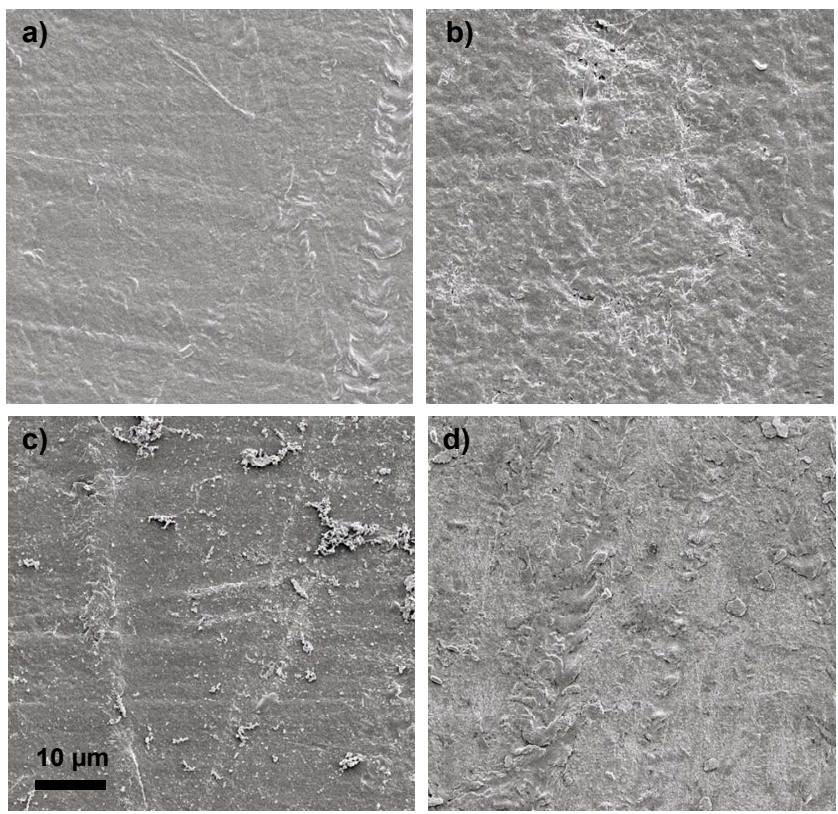

Figure S11. SEM micrographs of PBSeb ${ }_{90} \mathrm{Glux}_{10}$ : initial sample (a); after incubation at $\mathrm{pH} 2$ for four weeks (b); after incubation at $\mathrm{pH} 7.4$ for four weeks without (c) and with (d) porcine pancreas lipase.

Table S1. Compared molecular weights of $\mathrm{PB}_{90} \mathrm{Glux}_{10} \mathrm{Seb}$ in different reaction conditions.

\begin{tabular}{|c|c|c|c|c|c|c|c|c|}
\hline \multirow{3}{*}{ 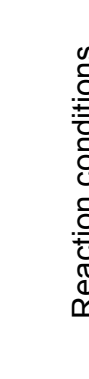 } & \multirow{3}{*}{ 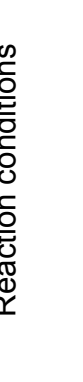 } & $\begin{array}{c}10 \% \text { wt } \\
\text { CALB vs } \\
\text { total amount }\end{array}$ & \multicolumn{6}{|c|}{$20 \%$ wt CALB vs total amount of monomers } \\
\hline & & $\begin{array}{l}\text { 1) } 90^{\circ} \mathrm{C} / 5 \mathrm{~h} \\
\text { 2) } 95^{\circ} \mathrm{C} / 72 \mathrm{~h}\end{array}$ & & \multicolumn{2}{|c|}{$\begin{array}{l}\text { 1) } 90^{\circ} \mathrm{C} / 24 \mathrm{~h} \\
\text { 2) } 90^{\circ} \mathrm{C} / 24 \mathrm{~h}\end{array}$} & \multirow{2}{*}{$\begin{array}{l}\text { 1) } \\
\text { 2) } \\
3 X\end{array}$} & $\begin{array}{l}90^{\circ} \mathrm{C} / 24 \mathrm{~h} \\
95^{\circ} \mathrm{C} / 72 \mathrm{~h}\end{array}$ & \multirow[b]{2}{*}{$7 X$} \\
\hline & & $5 X$ & $3 X$ & $5 X$ & $7 X$ & & $5 X$ & \\
\hline \multirow{3}{*}{ 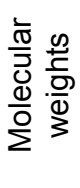 } & $M_{\mathrm{n}}$ & 6300 & 5200 & 5000 & 5100 & 5000 & 5100 & 5200 \\
\hline & $M_{\mathrm{w}}$ & 9100 & 6700 & 6500 & 6500 & 7000 & 6400 & 6400 \\
\hline & PD & 1.4 & 1.3 & 1.3 & 1.3 & 1.4 & 1.3 & 1.2 \\
\hline
\end{tabular}

GPC measurement was performed in THF solvent. 
Table S2. Thermal properties of some copolyesters obtained by enzymatic synthesis. ${ }^{\text {a }}$

\begin{tabular}{l|cccccccc}
\hline & $\begin{array}{c}T_{\mathrm{m} 1} \\
\left({ }^{\circ} \mathrm{C}\right)\end{array}$ & $\begin{array}{c}\Delta H_{\mathrm{m} 1} \\
\left(\mathrm{~J} \cdot \mathrm{g}^{-1}\right)\end{array}$ & $\begin{array}{c}T_{\mathrm{c}} \\
\left({ }^{\circ} \mathrm{C}\right)\end{array}$ & $\begin{array}{c}T_{\mathrm{m} 2} \\
\left({ }^{\circ} \mathrm{C}\right)\end{array}$ & $\begin{array}{c}\Delta H_{\mathrm{m} 2} \\
\left(\mathrm{~J} \cdot \mathrm{g}^{-1}\right)\end{array}$ & $\begin{array}{c}T_{\mathrm{d}} \\
\left({ }^{\circ} \mathrm{C}\right)\end{array}$ & $\begin{array}{c}T_{\mathrm{ds}} \\
\left({ }^{\circ} \mathrm{C}\right)\end{array}$ & $\begin{array}{c}\mathrm{RW} \\
(\%)\end{array}$ \\
\hline PB $_{92}$ Glux $_{8}$ Seb & $61(56)$ & $114(85)$ & $37(39)$ & $54(56)$ & $90(67)$ & $391(393)$ & $417(420)$ & $2(5)$ \\
PB $_{78}$ Glux $_{22}$ Seb & $50(49)$ & $69(64)$ & $18(23)$ & $47(45)$ & $59(49)$ & $389(393)$ & $419(419)$ & $2(5)$ \\
PBSeb $_{91}$ Glux $_{9}$ & $60(56)$ & $118(81)$ & $38(34)$ & $55(54)$ & $93(62)$ & $386(381)$ & $414(418)$ & $2(3)$ \\
PBSeb $_{73}$ Glux $_{27}$ & $42(43)$ & $61(45)$ & $19(-)$ & $38(35)$ & $50(26)$ & $286(356)$ & $413(419)$ & $7(3)$ \\
\hline
\end{tabular}

${ }^{a}$ In parenthesis, values obtained for chemically synthesized copolyesters of approximately similar compositions are given in parenthesis. See Table 4 in main document. 